Review

\title{
Research progress of natural products for the treatment of ischemic stroke
}

\author{
Jiaxin $\mathrm{Li}^{1, \dagger}$, Tingting Zhao ${ }^{2, \dagger}$, Hanzi Qiao ${ }^{3}$, Yanling Li ${ }^{1}$, Mengyao Xia ${ }^{1}$, Xiaoyu Wang ${ }^{1}$, Chuanguo Liu ${ }^{4}$, Tingting Zheng ${ }^{1}$, \\ Renjie Chen ${ }^{4}$, Yicheng $\mathrm{Xie}^{5}$, Jibiao Wu ${ }^{4}$, Xia Wei ${ }^{6}$, Jun Li ${ }^{6, *}$, Yu Feng ${ }^{1, *}$, Peng Sun ${ }^{4, *}$ \\ ${ }^{1}$ School of Pharmacy, Shandong University of Traditional Chinese Medicine, 250355 Ji'nan, Shandong, China \\ ${ }^{2}$ College of Foreign Languages, Shandong University of Traditional Chinese medicine, 250355 Ji'nan, Shandong, China \\ ${ }^{3}$ The Second Affiliated Hospital, Guangzhou University of Chinese Medicine, 510006 Cuangzhou, Guangdong, China \\ ${ }^{4}$ Innovation Research Institute of Chinese Medicine, Shandong University of Traditional Chinese medicine, 250355 Ji'nan, Shandong, China \\ ${ }^{5}$ The Children's Hospital, Zhejiang University School of Medicine, National Clinical Research Center for Child Health, 310030 Hangzhou, Zhejiang, China \\ ${ }^{6}$ Shandong Institute for Food and Drug Control, 250101 Jinan, Shandong, China \\ *Correspondence: sunpeng@sdutcm.edu.cn (Peng Sun); fyshandong@163.com (Yu Feng); 13573756821@163.com (Jun Li) \\ ${ }^{\dagger}$ These authors contributed equally.
}

\section{DOI:10.31083/j.jin2101014}

This is an open access article under the CC BY 4.0 license (https://creativecommons.org/licenses/by/4.0/).

Submitted: 31 May 2021 Revised: 2 July 2021 Accepted: 22 September 2021 Published: 28 January 2022

Stroke is a leading cause of death and disability world-widely. The incidence rate of stroke has been increasing due to the aging population and lifestyle changes. At present, the only drug approved by the US Food and Drug Administration (FDA) for the treatment of ischemic stroke is tissue plasminogen activator (t-PA), but its clinical application is greatly limited because of its narrow time window and bleeding risk. Natural products have a long history of being used in traditional medicine with good safety, making them an important resource for the development of new drugs. Indeed, some natural products can target a variety of pathophysiological processes related to stroke, including oxidative stress, inflammation and neuronal apoptosis. Therefore, the development of high-efficiency, lowtoxicity, safe and cheap active substances from natural products is of great significance for improving the treatment alternatives of patients with stroke. This article reviews the neuroprotective effects of 33 natural compounds by searching recent related literature. Among them, puerarin, pinocembrin, quercetin, epigallocatechin-3-gallate (ECCC), and resveratrol have great potential in the clinical treatment of ischemic stroke. This review will provide a powerful reference for screening natural compounds with potential clinical application value in ischemic stroke or synthesizing new neuroprotective agents with natural compounds as lead compounds.

\section{Keywords}

Natural products; Ischemic stroke; Mechanism

\section{Introduction}

Stroke is a major cause of death and disability in the world, often occurring in elderly patients [1]. There are two main types of stroke: ischemic (IS) and hemorrhagic stroke (HS). Among these, IS accounts for $80 \%$ of all cases of stroke and is caused by a blood clot in a cerebral artery. HS can be divided into intracerebral hemorrhage and subarachnoid hemorrhage and accounting for approximately $20 \%$ of the total number of stroke cases [2,3]. With unhealthy dietary pat- terns, increased prevalence of high blood pressure and an aging population, the incidence rate of stroke has been increasing over recent years, bringing a heavy burden to society, families and individuals, therefore becoming a global public health issue $[4,5]$.

At present, the two therapeutic strategies for stroke are reperfusion and neuroprotection [6]. Reperfusion includes mechanical thrombectomy and thrombolytic therapy. However, due to this short time window of reperfusion therapy, most patients do not receive effective treatment in time. In addition, thrombolytic therapy also has the risk of reperfusion injury and bleeding, with poor long-term efficacy $[7,8]$. The development of neuroprotective drugs mainly includes calcium channel antagonists, free radical scavengers, glutamate antagonists and cell membrane stabilizers, all of which are currently in the experimental stage [9]. Therefore, the characterization of novel drugs that protect against ischemic and neuronal damage is an important task at present.

Natural products have received extensive attention in recent years as a valuable resource bank for drug discovery due to their rich resources and long history of clinical application [10]. Indeed, many natural compounds against stroke have been found. They have a significant role in the early intervention of stroke, reducing adverse reactions, thus becoming potentially effective for the prevention and treatment of stroke $[11,12]$. Some natural compounds can simultaneously target multiple cell signaling pathways to regulate cell function. Considering the complexity of stroke pathophysiology, multi-target strategy should be more effective for the treatment of ischemic stroke [13]. In this paper, the mechanism of different natural products in the treatment of stroke was reviewed to provide a reference for further development of drugs for the prevention and treatment of stroke. 


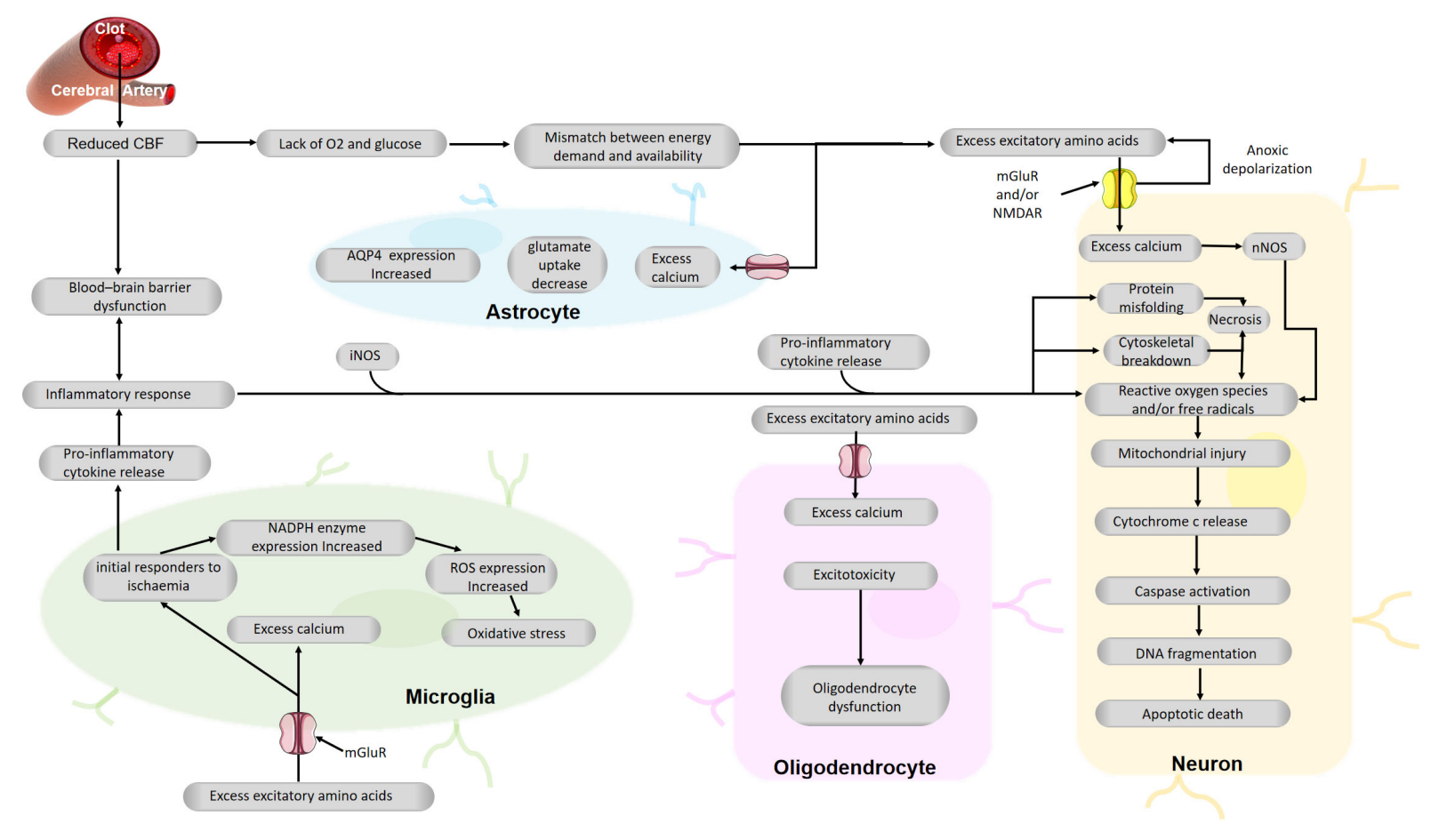

Fig. 1. Pathophysiology of stroke.

\section{Methodology}

A systematic literature search through 2000-2021 was performed in Google Scholar, PubMed, and a Scientific Direct Database to identify relevant studies. We used natural compounds, phytochemicals, polyphenols, flavonoids, quercetin, kaempferol, curcumin and other search terms in the full text options, and combined stroke, cerebrovascular accident, cerebral ischemia, and ischemic stroke. The titles and abstracts were scanned to exclude any studies that were clearly irrelevant. We read the full texts of the remaining articles and classified the compounds according to their chemical structures.

\section{Pathological mechanism of stroke}

Stroke is a series of complicated pathophysiological reactions caused by ischemia and hypoxia. It is currently believed that energy metabolism disorders, cytotoxic effects of excitatory amino acids, oxidative stress injury, inflammation and apoptosis are the main mechanisms that cause neuronal death in focal ischemic areas of the brain and lead to IS (Fig. 1) [14].

Cerebral artery occlusion leads to the decrease of cerebral blood flow, the insufficiency of oxygen and glucose supply, the disorder of energy metabolism in the brain, and the mismatch between energy requirements and availability of neurons, glial cells and endothelial cells. Anoxic depolarization and decreased glutamate reuptake activity lead to the increase of extracellular glutamate level, activation of glutamate receptor, promotion of the influx of calcium ions into cells, mitochondrial dysfunction and a series of signal events such as the activation of free radicals and proteases, and neu- ronal apoptosis. This process has been termed excitotoxicity. Excessive extracellular excitatory amino acids can inhibit the absorption of cysteine by oligodendrocytes, resulting in dysfunction of oligodendrocytes. Blood-brain barrier dysfunction and release of signaling molecules (for example, cytokines) from microglia lead to an inflammatory response. Inflammatory response promotes the release of inducible nitric oxide synthase (iNOS) from neutrophils, leading to the increase of nitric oxide level. Activated microglia can increase the expression of NADPH oxidase, induce ROS production, cause oxidative stress and further damage.

\subsection{Disturbance of energy metabolism in the brain}

The disturbance of energy metabolism in the brain is a direct factor that contributes to brain injury in the early stages of IS. The brain tissue is the most oxygen-consuming in the body, therefore ischemia can easily disturb energy metabolism, reduce blood flow, limit the supply of oxygen and glucose, and reduce the production of ATP in mitochondria $[15,16]$. ATP deficiency leads to the inhibition of ATPdependent $\mathrm{Na}^{+} / \mathrm{K}^{+}$-ATP pump and $\mathrm{Ca}^{2+}$-ATP pump on the cell membrane as well as $\mathrm{Na}^{+}$influx and $\mathrm{K}^{+}$efflux, resulting in the depolarization of the nerve cell membrane, excessive $\mathrm{Ca}^{2+}$ influx, and activation of phospholipases and membrane proteases. Ultimately, these reactions will damage the cell membrane, induce swelling and necrosis of the brain tissue, and eventually culminate in irreversible brain damage [17].

\subsection{Toxic effects of excitatory amino acids (EAA)}

Glutamic acid (Glu) and aspartic acid (Asp) are the two main excitatory amino acid transmitters in the central ner- 
vous system, of which Glu is the most abundant in the brain and is mainly distributed in the cortex and hippocampus $[18,19]$. When the blood supply to the brain is insufficient, Glu is released in large quantities from presynaptic nerve endings, decreasing the ability of neurons and astrocytes to absorb Glu, which will disrupt the dynamic balance of Glu synthesis and decomposition, uptake and release. Glu accumulates in the synaptic space and over activates its receptor, which can cause a series of reactions such as calcium overload, increased production of nitric oxide, and generation of free radicals, which are highly toxic to the central nervous system, exerting an improvisational neurotoxic effect, namely excitotoxic effects [20,21]. In addition, excessive extracellular EAA can inhibit the absorption of cysteines by oligodendrocytes, thereby reducing the synthesis of glutathione and damaging to oligodendrocytes [22].

\subsection{Oxidative stress}

Upon cerebral ischemia, the mitochondrial respiratory chain is damaged and ATP synthesis is reduced, which is accompanied by the excessive generation of reactive oxygen species (ROS) and reactive nitrogen species (RNS) [23]. In addition, activated microglia can increase the expression of nicotinamide adenine dinucleotide phosphate (NADPH) oxidases, inducing the generation of ROS [24, 25]. ROS has been reported to accumulate in ischemic tissue; its strong oxidation ability leads to the peroxidation of lipids, proteins and unsaturated fatty acids on the basement membrane and destroys the blood-brain barrier (BBB), ultimately causing irreversible damages on nucleic acid and carbohydrate metabolism [26, 27]. Oxidative stress also indirectly affects the permeability of the BBB by promoting the cytoskeleton reorganization, regulating the expression and distribution of tight junction proteins, affecting the expression and activity of matrix metalloproteinases, and promoting the synthesis of inflammatory factors. In the ischemic core, severe oxidative stress can lead to cell necrosis by degrading key cell components, while in the ischemic penumbra, oxidative stress activates NF- $\kappa$ B and MAPK signaling pathways to induce apoptosis.

\subsection{Inflammation}

The inflammatory response after cerebral ischemia plays an important role in the occurrence and development of IS, and a variety of immune cells and inflammatory cytokines participate in this process. During cerebral ischemia, activated astrocytes and microglia produce tumor necrosis factor- $\alpha$ (TNF- $\alpha$ ), platelet activating factor (PAF), interleukin-1 $\beta$ (IL-1 $\beta$ ), interleukin-6 (IL-6) and Interferon$\gamma($ IFN- $\gamma)[28-30]$. On the one hand, these inflammatory cytokines recruit immune cells, promote signal cascade reactions, increase the production of stimulating signals such as free radicals, and chemokines, and aggravate the inflammatory response. However, on the other hand, inflammatory cytokines induce endothelial cells, astrocytes, microglia, monocytes and macrophages to synthesize matrix metallo- proteinases such as matrix metalloproteinase (MMP)-2 and MMP-9, thereby destroying the basement membrane and tight junction proteins. This process increases the permeability of the BBB, and can cause cerebral edema, cerebral hemorrhage and neuronal injury [31]. After ischemic injury, the expression of inflammation-related genes BRAHMA (BRM), $\mathrm{I} \kappa \mathrm{B}$ (also known as NF $\kappa \mathrm{B}$ inhibitor), FOXF1 and ITIH- 5 in the stroke vasculome is upregulated, which promotes and aggravates the progress of stroke [32].

\subsection{Neuronal apoptosis}

Within a few hours after cerebral ischemia, dysfunctional energy metabolism, excitotoxicity of EAA, free radical damage and inflammatory immune effects will eventually induce neuronal cell death or apoptosis around the infarcted area or ischemic penumbra [33]. According to different forms of activation, apoptosis can be divided into the exogenous death receptor signaling pathway and endogenous signaling pathway, involving the activation of a variety of genes and proteins. In the endogenous pathway, the protein Bax forms oligomeric channels on the mitochondrial membrane, leading to the outflow of cytochrome $\mathrm{c}$, which activates caspase 3 , and ultimately removes cytoskeletal proteins and induces DNA cleavage [34-36]. As an important tumor suppressor protein and transcription factor, p53 can directly induce the expression of apoptotic proteins and genes under ischemia and hypoxia [37]. Neuronal death caused by ischemia commonly results in cell necrosis and apoptosis. Lack of oxygen induces the opening of calcium channels, and this large influx of $\mathrm{Ca}^{2+}$ regulates the expression of the protein Bid in the apoptotic pathway by activating calpain, and finally promoting the activation of the apoptotic pathway [38]. After focal ischemia-reperfusion injury, irreversible damage occurs in the ischemic center, while the neuronal apoptosis that occurs in the ischemic penumbra is reversible. Therefore, reducing neuronal apoptosis in the ischemic penumbra is an effective method for the treatment of stroke [39].

\subsection{Other mechanisms}

The blood-brain barrier (BBB) plays a vital role in regulating the trafficking of fluid, solutes and cells at the blood-brain interface and maintaining the homeostatic microenvironment of the CNS [40]. Pathological mechanisms such as inflammation and oxidative stress caused by cerebral ischemia and hypoxia can change the permeability of the BBB, resulting in the permeability towards water molecules and ions, which can form brain edema [41]. In addition, the expression of aquaporin 4 (AQP4) on the astrocyte foot process is up-regulated after cerebral ischemia-reperfusion, which can also allow excess water to accumulate in the brain [42, 43]. As a serious complication of stroke, cerebral edema can lead to higher intracranial pressure, compression of brain tissue, obstruction of cerebrospinal fluid circulation and blood flow, aggravating ischemia and edema, thus forming a vicious cycle [44, 45]. 


\section{Natural products for ischemic stroke}

Many natural compounds that can treat stroke have been well studied (Figs. 2 (Ref. [46-91]), 3 (Ref. [92-96]), 4 (Ref. [97-101]), 5 (Ref. [102-104]), 6 (Ref. [105, 106]), 7 (Ref. [107-111])). We reviewed and summarized them for future drug discovery and application (Table 1, Ref. [46, 49, 5254, 56, 58-63, 66, 68-70, 72-74, 76, 77, 79-81, 83, 85, 87, 90, 91, 94-98, 100, 102-111]).

\subsection{Polyphenols}

Polyphenols exist in many medicinal and edible plants, and possess a wide range of beneficial effects against atherosclerosis, brain dysfunction, stroke and cardiovascular diseases [112]. Polyphenols are molecules chemically characterized by the presence of at least one aromatic ring with one or more hydroxyl groups attached [113]. According to its chemical structure, polyphenols can be divided into flavonoids, lignans, stilbenes, curcuminoids and so on [2].

\subsubsection{Flavonoids}

Bioflavonoids are well-known antioxidants with neuroprotective effects. For instance, puerarin is the main active component of Puerariae Lobatae Radix and can regulate neuronal autophagy of rats subjected to cerebral ischemiareperfusion injury by activating the APMK-mTOR-ULK1 signaling pathway [46]. Puerarin treatment was shown to significantly reduce neuronal autophagy in the infarct penumbra of middle cerebral artery occlusion (MCAO) rats, and reduced cerebral infarction volume, cerebral edema and neurological deficits [47]. Meta analysis of 35 randomized controlled trials showed that puerarin injection can improve neurological deficit and reduce blood viscosity in patients with cerebral ischemia, and its clinical effective rate is better than aspirin [48].

Pinocembrin $(\mathrm{PCB})$ is a natural flavonoid found in propolis, but also found in pepper and compositae. Administration of PCB 5 minutes before t-PA can significantly reduce ischemic BBB injury and cerebral infarction volume, reduce cerebral edema, improve survival rate, and extend the time window of t-PA treatment [49]. Other studies have shown that PCB can reduce the protein expression levels of light chain 3- II (LC3II) and Beclin1 in the hippocampal CA1 region of rats subjected to brain ischemia-reperfusion injury, increasing the level of p62, alleviating neuronal injury and cognitive decline, and reducing autophagy induced by autophagy inducer rapamycin (RAPA) [50]. Given its significant efficacy in cerebral ischemia, pinocembrin has been approved by China Food and Drug Administration (CFDA) as a new treatment drug for ischemic stroke and is currently in progress of phase II clinical trials [51].

Baicalin is a natural flavonoid found in the dried root of Scutellaria baicalensis Georgi. Treatment with baicalin can reduce the levels of peroxynitrite anion (ONOO-) in ischemic brain tissue, inhibit the activation of MMP-9 and reduce the expression of AQP4 in the brain. Baicalin combined with t-PA can expand the therapeutic window of $t-$
PA, reduce mortality, alleviate BBB injury and brain edema. Moreover, this flavonoid can be used as a potential candidate for t-PA combination therapy [52]. Baicalin also decreased intracellular calcium concentration and phosphorylation of $\mathrm{Ca}^{2+} /$ calmodulin-dependent protein kinase II (CaMKII) in hippocampal neurons induced by oxygen-glucose deprivation (OGD), thus reducing the apoptosis of hippocampal neurons and SH-SY5Y cells [53].

Quercetin is a flavonol widely distributed in flowers, leaves, and fruits of different species of plants. It is an active ingredient in traditional Chinese medicines such as $\mathrm{Bu}$ pleurum chinensis DC., Ginkgo biloba Linn. and Folium Mori. Quercetin treatment can down-regulate the expression of caspase- 3 and poly ADP-ribose polymerase (PARP), reduce the cerebral infarction volume of focal cerebral ischemia animal models, decrease neuronal apoptosis, inhibit the activity of myeloperoxidases (MPO) in ischemic brain tissue and reduce the nerve injury caused by oxidative stress $[54,55]$. In addition, quercetin is neuroprotective due to its modulation of paralbumin expression during ischemic brain injury [56]. 4-methylcatechol is a human intestinal metabolite of quercetin, which is an effective platelet inhibitor. It has better curative effect than the commonly used antiplatelet drug acetylsalicylic acid, which supports its application in the prevention of thrombotic stroke [57].

Eriodictyol is a flavonoid isolated from Dracocephalum rupestre Hance. In a rat permanent middle cerebral artery occlusion (pMCAO) model, elycohol was shown to inhibit the expression of MPO, decrease the inflammatory response, and down-regulate the expression of TNF- $\alpha$ and inducible nitric oxide synthase (iNOS) in the ischemic cortex. This ultimately resulted in reduced infarct size and memory deficit as well as an improved motor function [58]. Eriodictyol pretreatment can down-regulate the expression of TNF- $\alpha$, IL6 , IL- 10 and IL- $\beta 1$ in the serum and cerebral cortex of temporary MCAO (tMCAO) rats, and inhibit the expression of apoptosis-related proteins Bax and cleaved-caspase-3, indicating that eriodictyol can reduce the inflammatory response after brain injury and inhibit neuronal apoptosis and improve neurological function, which may be partly attributed to the reversal of the protective effect of autophagy [59].

Isorhamnetin is a flavonoid isolated from ginkgo, seabuckthorn and other medicinal plants. In an experimental rat model of MCAO and reperfusion, isorhamnetin was able to reduce the activities of caspase- 3 and MPO, alleviate oxidative stress, improve the recovery of neuronal function, reduce brain edema, and up-regulate the expression of tight junction proteins $\mathrm{ZO}-1$ and claudin -5 to protect the integrity of BBB [60].

Apigenin is a natural flavonoid that mainly exists in celery, parsley, chamomile and other plants. Apigenin can inhibit the expression of histone deacetylase (HDAC), induce the expression of brain-derived neurotrophic factor (BDNF) and synapsin I (Syn-I) mRNA as well as reduce the cognitive impairment after cerebral ischemia-reperfusion injury in 


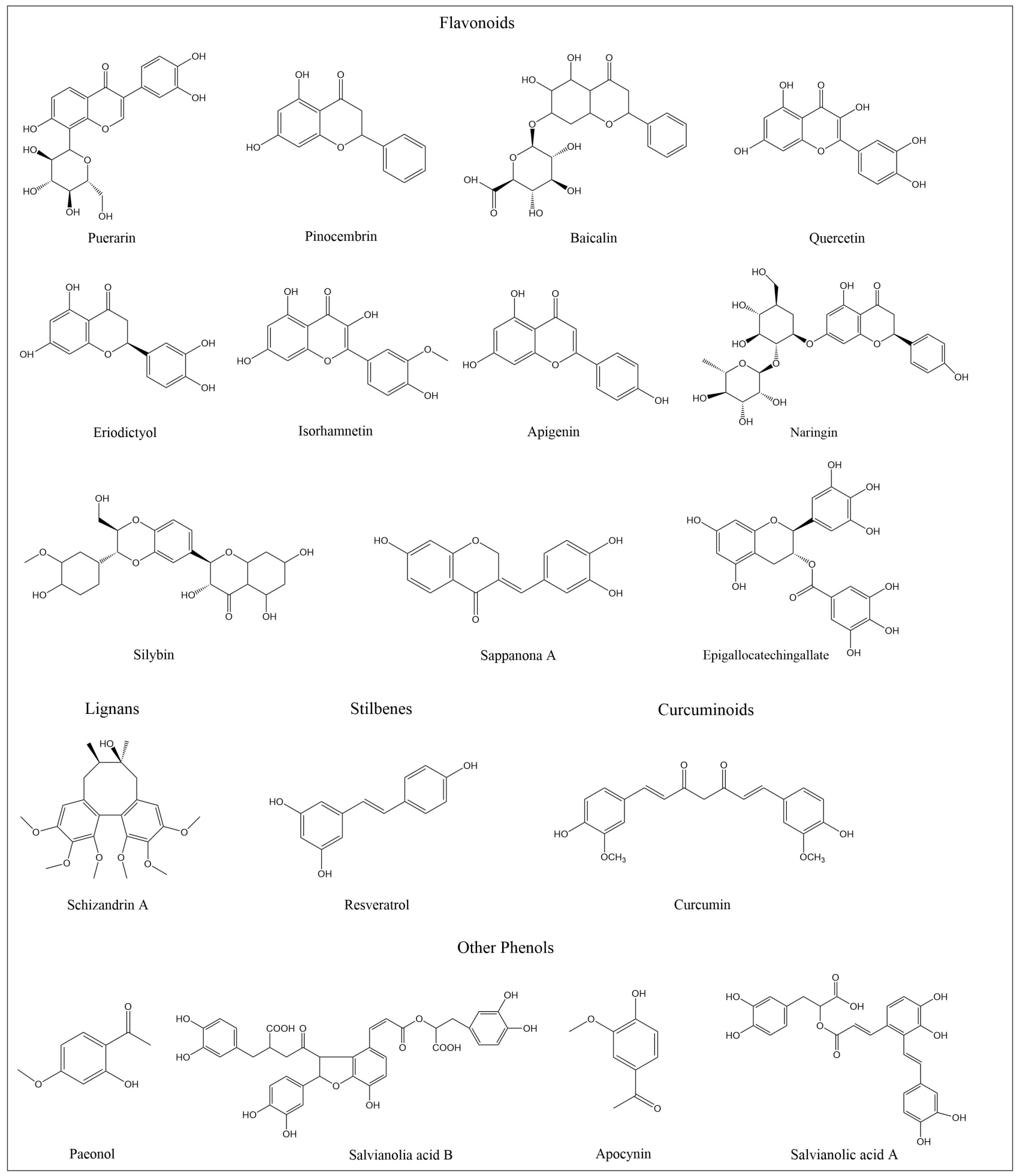

Fig. 2. Chemical structures of some representative neuroprotective phenols (Ref. [46-91]).

rats [61]. Apigenin can improve the neurological function of cerebral ischemia-reperfusion rats by up-regulating the Caveolin-1/VEGF pathway, and reduce pathological changes and cerebral infarction volume, and promote the proliferation of vascular endothelial cells, thereby having a protective effect on cerebral ischemia-reperfusion injury [62].
Naringin is derived from the immature or near-ripe dry peels of rutaceae plants pomelo and grapefruit. Naringin has a strong $\mathrm{ONOO}^{-}$scavenging ability and can inhibit $\mathrm{ONOO}^{-}$-mediated mitosis, NADPH oxidase, iNOS, NO production, and protect against ischemia-reperfusion injury [63]. In addition, naringin can reduce neuronal death and 


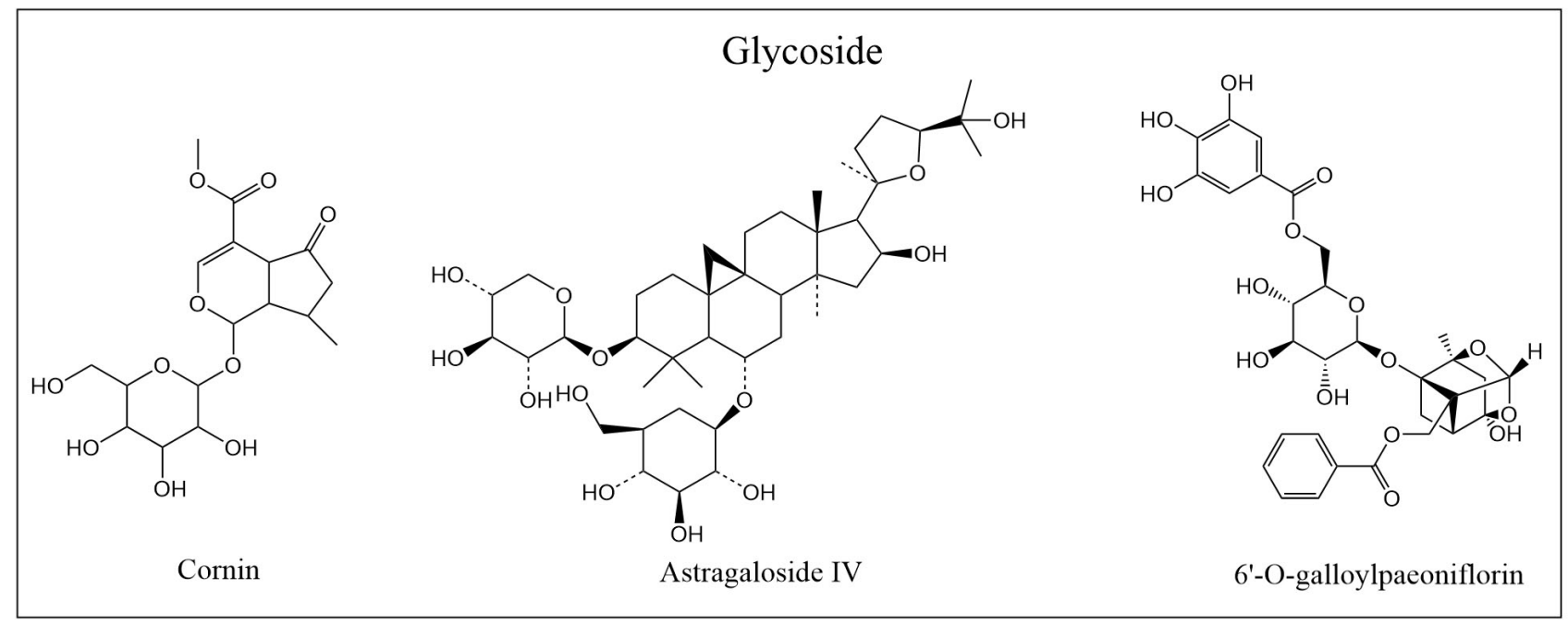

Fig. 3. Chemical structures of some representative neuroprotective glycosides (Ref. [92-96]).

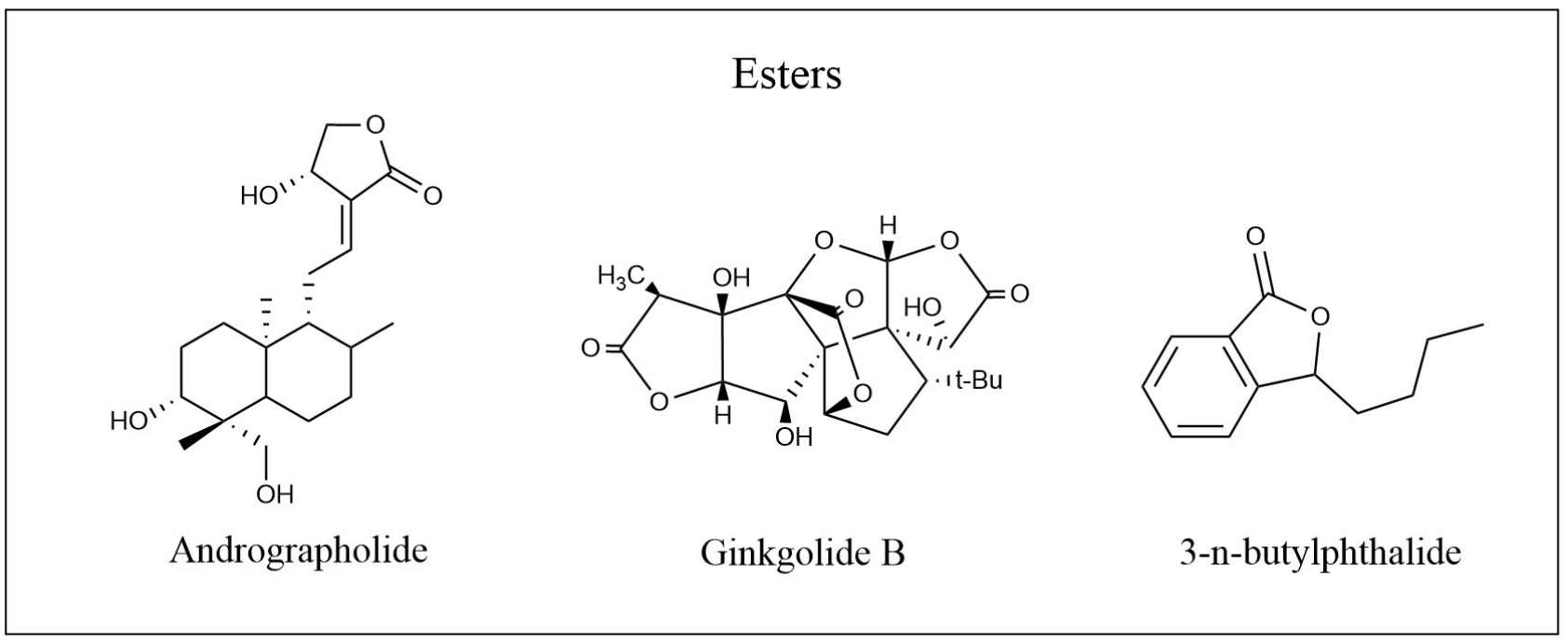

Fig. 4. Chemical structures of some representative neuroprotective esters (Ref. [97-101]).

inhibit the over activation of astrocytes and microglia after ischemic injury in tMCAO mice $[64,65]$. In the OGD model of neuronal cells, naringin pretreatment can activate the PI3K/AKT pathway, improve cell viability, and inhibit the secretion of inflammatory factors such as TNF- $\alpha$ and IL6 [66].

Silymarin is a natural flavonoid extracted from the dried fruit of Silybum marianum (L.) Gaertn, and its main components are silybin, isosilybin, silydianin and silychristin, among which silybin has the highest concentration and the strongest activity [67]. In a pMCAO rat model, silymarin was shown to inhibit apoptosis by modulating procaspase-3 level and balancing the expression of B-cell lymphoma-2 (Bcl-2) and Bax, as well as through the inhibition of autophagy by reducing the levels of LC3-II and Beclin1, thus exerting a neuroprotective effect [68].
Sappanone A is a natural high isoflavone in the legume plant Caesalpinia sappan L., which has significant antiinflammatory and antioxidant effects. Sappanone A pretreatment can down-regulate the levels of CCAAT/enhancer binding protein homologous protein (CHOP) and glucose regulatory protein 78 (GRP78) in tMCAO rats, inhibit endoplasmic reticulum stress, reduce inflammation, oxidative stress and apoptosis, and alleviate neuronal injury after cerebral ischemia-reperfusion injury [69].

Epigallocatechin-3-gallate (EGCG) is the main component of polyphenols contained in green tea. EGCG can activate $\mathrm{PI} 3 \mathrm{~K} / \mathrm{Akt} / \mathrm{eNOS}$ signaling pathway, down-regulate the expression of caspase- 3 and Bax, increase the expression of $\mathrm{Bcl}-2$, reduce neuronal apoptosis and protect against brain injury induced by pMCAO [70]. In clinical trials, EGCG supplementation during rt-PA therapy can prolong the time window of rt-PA treatment, significantly reduce the adverse 


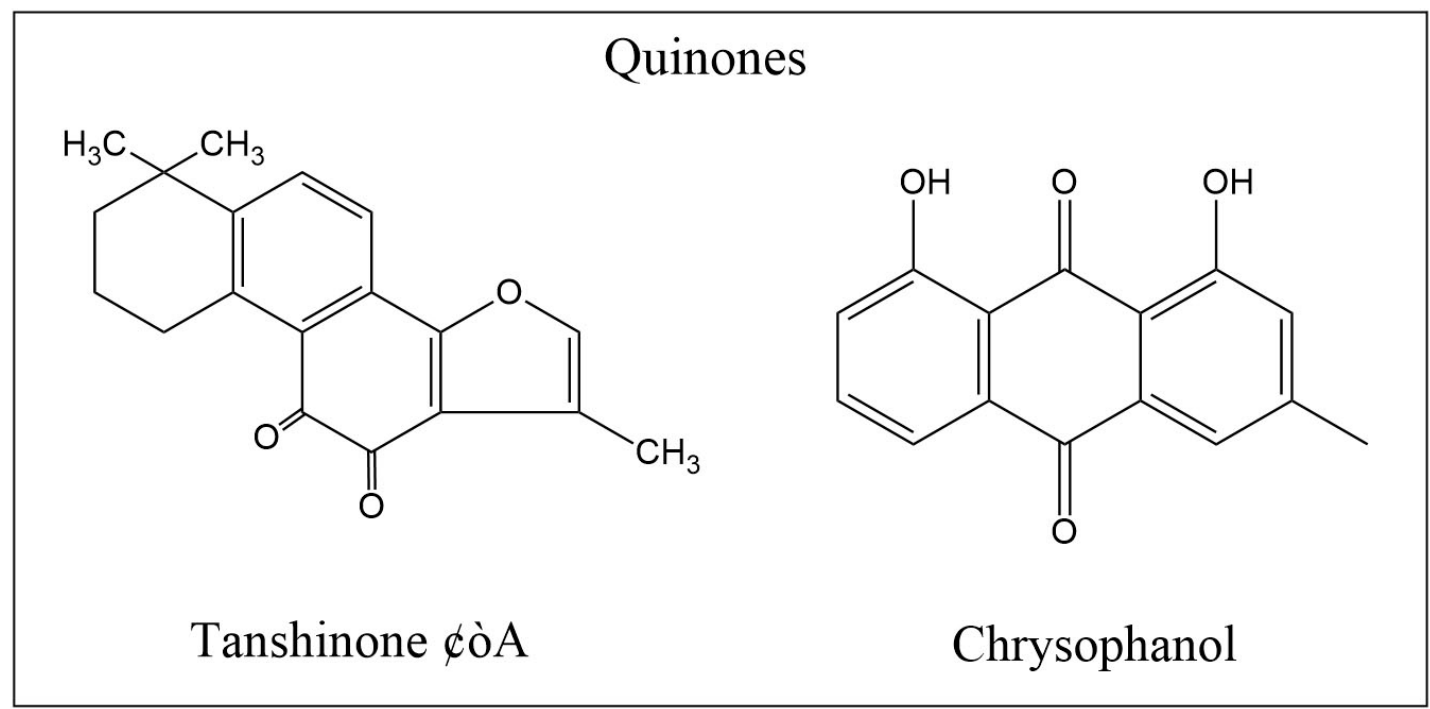

Fig. 5. Chemical structures of some representative neuroprotective quinones (Ref. [102-104]).

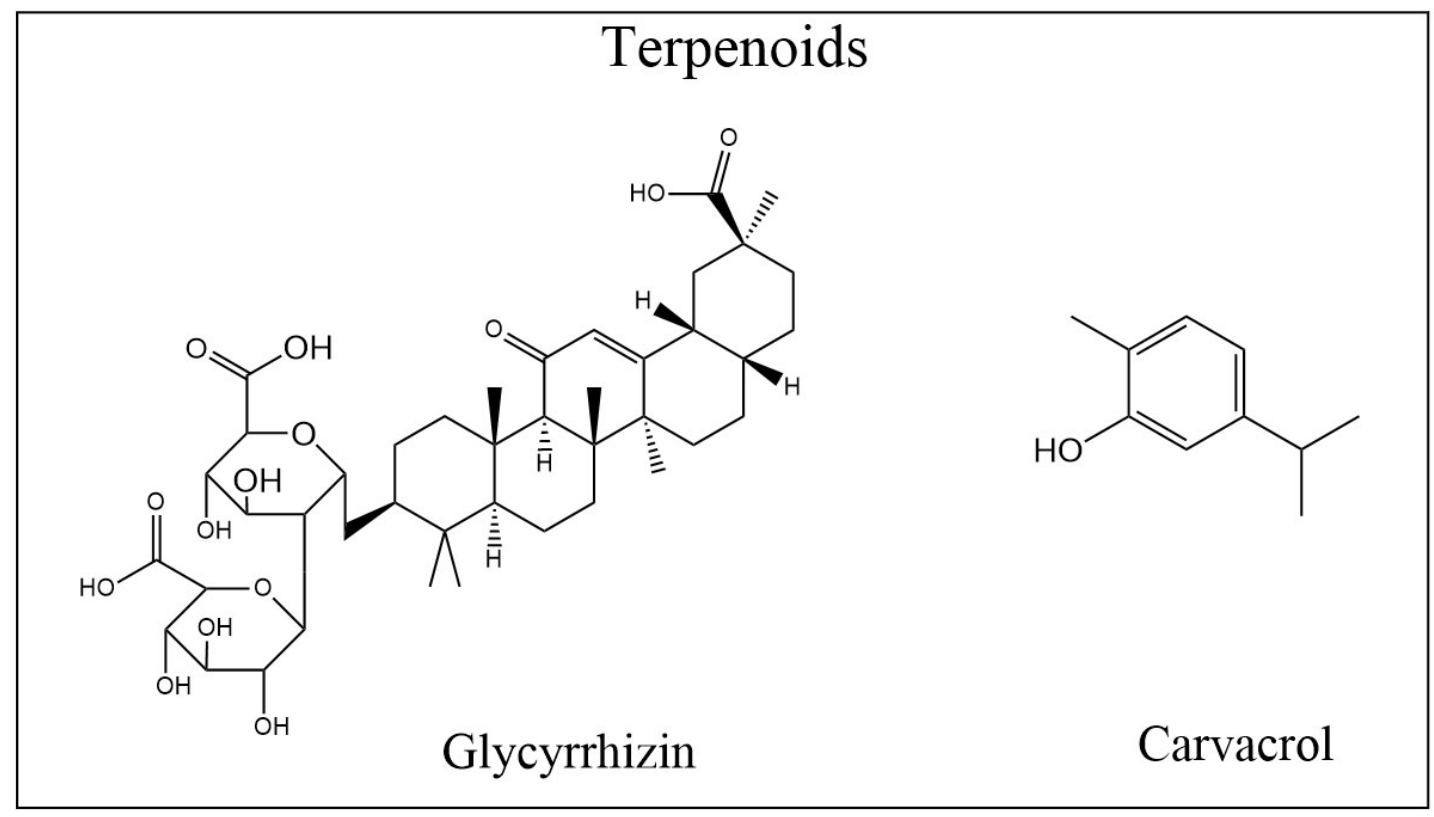

Fig. 6. Chemical structures of some representative neuroprotective terpenoids (Ref. [105, 106]).

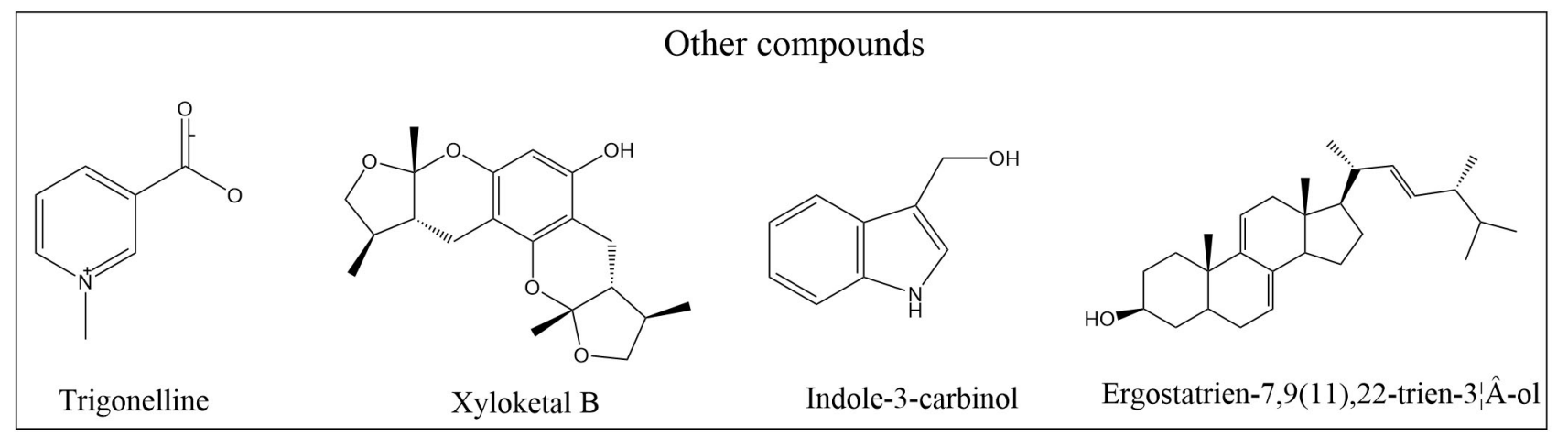

Fig. 7. Chemical structures of some representative neuroprotective compounds (Ref. [107-111]). 
reaction of delayed administration of rt-PA and reduce the infarct volume and destruction of BBB. The underlying mechanism of EGCG in stroke involves the downregulation of MMP-2 and MMP-9 [71]. Other studies have shown that EGCG can promote angiogenesis, increase vascular density and reduce oxidative stress in tMCAO mice, which may be related to the up regulation of Nrf2 signaling pathway [72].

\subsubsection{Lignans}

Lignans are plant secondary metabolites derived from the oxidative coupling of phenylpropanoids [73]. Some lignans have been suggested to have potential in the prevention of cardiovascular disease and, possibly, stroke [74]. Schizandrin A is a lignin compound isolated from Schisandra chinensis, which can inhibit autophagic death of neurons after cerebral ischemia-reperfusion injury by regulating the AMPK-mTOR pathway, increasing the survival rate of PC12 cells after $\mathrm{OGD} / \mathrm{R}$, and increase mechanistic target of rapamycin (mTOR) phosphorylation [75]. Tail vein injection of Schizandrin A can improve the neurological function of MCAO rats, reduce infarct volume, increase SOD and CAT activities, reduce ROS production, down-regulate the transcription of COX-2 and iNOS, reduce the inflammatory cytokines IL- $1 \beta$, IL- 6 and TNF- $\alpha$, and increase the release of anti-inflammatory cytokines transforming growth factor- $\beta$ (TGF- $\beta$ ) and IL-10 [76].

\subsubsection{Stilbenes}

Stilbenes are natural phenolic defence compounds occurring in a number of different plant species that possess a wide range of beneficial activities, including the capability to protect against oxidative stress [77]. Resveratrol is a polyphenolic compound found in Cassia tora Linn., Veratrum nigrum Linn., Reynoutria japonica Houtt. and other medicinal plants. Studies have shown that the protective effect of resveratrol in IS is related to the regulation of the brain-gut axis [78]. Resveratrol can regulate the balance of T-helper 17/regulatory T cells (Th17/Tregs) and T-helper 1/T-helper 2(Th1/Th2) in intestinal lamina propria and the expression of proinflammatory cytokines (IL-17A, IL-23, IL-10, IFN$\gamma$ and IL-4) in the small intestine after MCAO. Resveratrol can also reduce the permeability of the intestinal epithelium and blood vessels, reduce the transfer of cytokines from the small intestine to the blood, and reduce neuroinflammation after stroke [79]. In addition, this polyphenol can improve the activities of glutathione peroxidase (GPx), glutathione reductase (GR), glutathione-S-transferase (GST), superoxide dismutase (SOD) and catalase (CAT) in the penumbra cortex, restore the integrity of the cell membrane and the activity of $\mathrm{Na}^{+} \mathrm{K}^{+}$ATP-ase, thus reducing infarct volume and improving the neurobehavioral functions in a rat $\mathrm{tMCAO}$ model [80]. Stroke patients received allopathic therapy and long-term resveratrol supplementation. After 12 months, compared with patients who only received allopathic therapy, patients in the resveratrol supplement group had signif- icantly lower blood pressure, body mass index, blood lipid parameters, and blood sugar levels. Importantly, there was no new vascular disease occurring in the patients, indicating that resveratrol as an adjuvant may play a role in the secondary prevention of stroke [81].

\subsubsection{Curcuminoids}

Curcumin is the main active component of Curcuma longa L., curcumin treatment can activate specific protein 1 (SP1), up-regulate the expression of peroxiredoxin 6 (RDX6) after 24 hours of cerebral ischemia and reperfusion injury, increase the number of PRDX6-positive neurons, reduce oxidative stress and exert a neuroprotective role [82]. Curcumin can alleviate the cognitive impairment caused by bilateral common carotid arteries occlusion (BCCAO), promote the proliferation of neural stem cells, promote the differentiation maturation of newly generated neural cells into neurons, increase the expression of neurogenesis related proteins (including Ngn2, Pax6 and NeuroD 1) and up regulate Wnt/ $\beta$ Catenin signaling pathway [83].

\subsubsection{Other phenols}

Paeonol is an active ingredient present in the root bark of Paeonia suffruticosa Andr. (Ranunculaceae) and the root or whole herb of Cynanchum paniculatum (Bunge) Kitagawa (Asclepiadaceae). Studies have shown that paeonol treatment can significantly reduce behavioral disorder, cerebral infarction volume and brain edema after 72 hours of ischemiareperfusion injury, as well as alleviate the neuronal damage in ischemic core and boundary areas after 28 days of reperfusion [84].

Salvianolic acid B (Salb) is a water-soluble component extracted from Salvia miltiorrhiza Bge. Salb is the most abundant and bioactive compound in salvianolic acid, accounting for approximately $70 \%$ of its content [85]. In a tMCAO model, SalB was shown to improve cerebral blood flow, reduce platelet aggregation, inhibit EAA neurotoxicity, reduce free radicals and improve energy metabolism. These effects were linked with inhibition of astrocytes and microglia, to prevent brain injury and promote the recovery of the motor function after cerebral ischemia/reperfusion injury in rats [86].

Apocynin is an o-methoxyphenol compound isolated from the rhizomes of Picrorhiza scrophulariiflora Pennell. Apocynin can reduce NADPH oxidase activity and ROS production after cerebral ischemia in rats, thereby preventing the activation of glial cells, down-regulating inflammation and pro-apoptotic markers, and alleviating mitochondrial injury after cerebral ischemia injury in rats $[87,88]$.

Salvianolic acid A (SAA) is a water-soluble phenolic acid isolated from the roots of Salvia miltiorrhiza Bge. SAA can significantly reduce the cerebral infarct area and brain water content of tMCAO rats, reduce neurological deficits and pathological changes, and enhance the anti-inflammatory and antioxidant capacities of tMCAO rats [89]. SAA was 
Table 1. Natural anti-Stroke chemicals.

\begin{tabular}{|c|c|c|c|c|c|c|}
\hline Category & Compound & Origin & Mode of study & Experimental model & Possible mechanisms & References \\
\hline \multirow[t]{18}{*}{ Polyphenols } & Puerarin & Puerariae lobatae Radix & In vivo & tMCAO & APMK-mTOR-ULK1 pathway activation & [46] \\
\hline & Pinocembrin & Propolis & In vivo & I/R injury & PDGF-CC/PDGFR $\alpha$ pathway activation; LC3II and Beclin1 & [49] \\
\hline & Baicalin & Scutellaria baicalensis Georgi & In vitro; in vivo & tMCAO, OGD & $\begin{array}{l}\text { ONOO-MMP-9 pathway inhibition; AQP4 inhibition; } \\
\text { p-CaMKII inhibition }\end{array}$ & {$[52,53]$} \\
\hline & Quercetin & - & In vivo & pMCAO & caspase- 3 and PARP inhibition; parvalbumin up-regulation & {$[54,56]$} \\
\hline & Eriodictyol & Dracocephalum rupestre Hance & In vivo & pMCAO, tMCAO & $\begin{array}{l}\text { TNF- } \alpha \text { and iNOS reduction; Bax and cleaved-caspase-3 } \\
\text { inhibition; Bcl-2 up-regulation }\end{array}$ & {$[58,59]$} \\
\hline & Isorhamnetin & Hippophae rhamnoides & In vivo & tMCAO & caspase- 3 and MPO inhibition & [60] \\
\hline & Apigenin & - & In vivo & tMCAO & HDAC inhibition; BDNF and Syn-I up-regulation; & {$[61,62]$} \\
\hline & Naringin & Citrus kawachiensis & In vitro; in vivo & $\mathrm{OGD} / \mathrm{RO}, \mathrm{tMCAO}$ & $\begin{array}{l}\text { Caveolin-1/VEGF pathway activation } \\
\text { ONOO }^{-} \text {reduction; NADPH oxidase inhibition; iNOS and } \\
\text { 3-NT reduction; PI3K/AKT pathway activation }\end{array}$ & {$[63,66]$} \\
\hline & silymarin & Silybum marianum (L.) Gaertn & In vivo & pMCAO & Bcl-2 up-regulation; Bax inhibition & [68] \\
\hline & Sappanone A & Caesalpinia sappan $\mathrm{L}$. & In vivo & tMCAO & CHOP and GRP78 reduction & [69] \\
\hline & Epigallocatechin-3-gallate & Green Tea & In vivo & pMCAO, tMCAO & PI3K/Akt/eNOS pathway activation; Nrf2 pathway activation & {$[70,72]$} \\
\hline & Schizandrin A & Schisandra dhinensis & In vitro; in vivo & $\mathrm{OGD} / \mathrm{R}, \mathrm{tMCAO}$ & $\begin{array}{l}\text { AMPK-mTOR pathway activitation; AMPK/Nrf2 pathway } \\
\text { activation }\end{array}$ & {$[73,74]$} \\
\hline & Resveratrol & - & In vivo & tMCAO & $\begin{array}{l}\text { IL-17A, IL-23, IL-10, IFN- } \gamma \text { and IL-4 reduction; GPx, GR, } \\
\text { GST, SOD, CAT and } \mathrm{Na}^{+} \mathrm{K}^{+} \text {ATPase up-regulation }\end{array}$ & {$[76,77]$} \\
\hline & Curcumin & Curcuma longa $\mathrm{L}$. & In vivo & tMCAO, BCCAO & $\begin{array}{l}\text { SP1/Prdx6 pathway activation; Wnt/ } \beta \text {-catenin pathway } \\
\text { activation; Ngn2, Pax6 and NeuroD1 up-regulation }\end{array}$ & {$[79,80]$} \\
\hline & Paeonol & Paeonia suffruticosa Andr & In vivo & tMCAO & $\begin{array}{l}\text { astrocytes proliferation reduction; microglial activation } \\
\text { inhibition }\end{array}$ & [81] \\
\hline & Salvianolic acid B & Salvia miltiorrhiza Bge. & In vivo & tMCAO & $\begin{array}{l}\text { ROS reduction; GFAP, Iba1, IL- } 1 \beta, \text { IL- } 6 \text {, TNF- } \alpha \text { and } \\
\text { cleaved-caspase } 3 \text { reduction }\end{array}$ & [83] \\
\hline & Apocynin & Picrorhiza scrophularififlora Pennell. & In vivo & tMCAO & NADPH oxidase inhibition, ROS reduction & [85] \\
\hline & Salvianolic acid A & Salviae Miltiorrhizae Bunge & In vivo & tMCAO & $\mathrm{ONOO}^{-}$reduction & [87] \\
\hline \multirow[t]{3}{*}{ Glycosides } & Cornin & Verbena officinalis $\mathrm{L}$. & In vitro; in vivo & $\begin{array}{l}\text { OGD, } \\
\text { tMCAO; }\end{array}$ & $\begin{array}{c}\text { Ang1/Tie2 axis activation; } \\
\text { Wnt/b-catenin pathways activation; PI3K-Akt-eNOS-VEGF } \\
\text { pathway activation }\end{array}$ & {$[90,91]$} \\
\hline & Astragaloside-IV & Astragalus membranaceus (Fisch.) Bunge. & in vivo & tMCAO & $\begin{array}{l}\text { death receptor pathway and mitochondrial pathway inhibition; } \\
\text { SIRT1 up-regulation; ac-MAPT and p-MAPT reduction }\end{array}$ & {$[94,95]$} \\
\hline & 6'-O-galloylpaeoniflorin & Paeonia lactiflora Pall. & In vitro; in vivo & OGD, tMCAO & $\mathrm{PI} 3 \mathrm{~K} / \mathrm{Akt} / \mathrm{Nrf2}$ pathway activation & [96] \\
\hline \multirow[t]{3}{*}{ Esters } & Andrographolide & Andrographis paniculata (Burm. f.) Nees. & In vivo & tMCAO & Nrf2-HO-1 pathway activation & [97] \\
\hline & Ginkgolide B & Ginkgo biloba $\mathrm{L}$. & In vitro & $\mathrm{OGD} / \mathrm{R}$ & Bcl-2 up-regulation; Bax inhibition & [98] \\
\hline & 3-n-butylphthalide & Apium graveolens $\mathrm{L}$. & In vivo & pMCAO & ATP degradation Inhibition & [100] \\
\hline
\end{tabular}


Table 1. Continued.

\begin{tabular}{|c|c|c|c|c|c|c|}
\hline Category & Compound & Origin & Mode of study & Experimental model & Possible mechanisms & References \\
\hline \multirow[t]{2}{*}{ Quinones } & Tanshinone IIA & Salvia miltiorrhiza Bge. & In vivo & pMCAO & PI3K/mTOR/HER-3 pathway activation & [102] \\
\hline & Chrysophanol & Rheum palmatum $\mathrm{L}$. & In vivo & tMCAO & $\begin{array}{l}\text { SOD and MnSOD activation; p-eIF } 2 \alpha-C H O P \text { pathway } \\
\text { inhibition }\end{array}$ & {$[103,104]$} \\
\hline \multirow[t]{2}{*}{ Terpenoids } & Glycyrrhizin & Glycyrrhiza uralensis Fisch. & In vivo & tMCAO & $\mathrm{ONOO}^{-} / \mathrm{HMGB} 1 / \mathrm{TLR} 2 / \mathrm{MMP}-9$ pathway inhibition & {$[105]$} \\
\hline & Carvacrol & Origanum dictamnus & In vivo & tMCAO & NF-kB pathway inhibition & [106] \\
\hline \multirow[t]{5}{*}{ Other compounds } & trigolineline & Trigonella foenum-graecum $\mathrm{L}$. & In vivo & tMCAO & MPO inhibition & {$[107]$} \\
\hline & Xycycline B & Xylaria sp. & \multicolumn{2}{|c|}{ In vitro; in vivo OGD, hypoxic-ischemice brain injury model } & caspase-3 reduction; Bax inhibition; Bcl-2 up-regulation & [108] \\
\hline & Indole-3-carbinol & brassica sp. & In vivo & tMCAO & SOD and CAT up-regulation & [109] \\
\hline & Ergostatrien-7,9(11),22-trien-3ß-ol & Antrodia camphorata & In vivo & tMCAO & PI3K/Akt pathway activation; GSK-3 inhibition & {$[110]$} \\
\hline & \multicolumn{4}{|c|}{ Achyranthes bidentate polypeptide $\mathrm{k}$ Adhyranthes Bidentata Bloom In vitro; in vivo } & $\begin{array}{l}\text { NF- } x \text { B pathway inhibition; TF and PAI-1 and MMP-2/-9 } \\
\text { inhibition }\end{array}$ & [111] \\
\hline
\end{tabular}


shown to improve the sensorimotor and persistent memory impairments caused by focal ischemia in the unilateral frontal cortex of rats, suggesting that SAA has therapeutic potential in the treatment of IS-related memory impairment [90]. In addition, SAA can inhibit the production of ONOO-, up-regulate the phosphorylation levels of protein kinase B (AKT), forkhead transcription factor (FKHR) and extracellular signal-regulated kinase (ERK), increase glucose metabolism after cerebral ischemia, reduce neurological deficits, and protect the brain from ischemia/reperfusion injury [91].

\subsection{Glycosides}

Cornin is an iridoid glycoside isolated from the fruits of Verbena officinalis L., which can induce angiogenesis in vitro by increasing the proliferation, invasion and lumen formation [92]. Subsequent studies confirmed that cornin can reduce BBB leakage and promote angiogenesis and functional recovery after stroke by increasing the proliferation of rat arterial smooth muscle cells (RASMC) and the expression of Wnt5a, $\beta$-catenin, cyclin D1 and angiopoietin-1(Ang1) [93].

Astragaloside IV(AS-IV), the main active component of Astragalus membranaceus (Fisch.) Bunge., was shown to exert neuroprotective effects in a rat cerebral ischemia-reperfusion injury model. It can modulate Fas, Fas ligand (FasL), caspase8 and $\mathrm{Bax} / \mathrm{Bcl}-2$, reduce the protein levels of apoptotic cytokines Bid, cleaved caspase-3 and Cytochrome C (Cyto $\mathrm{C})$, and reduce the volume of cerebral infarction and neuronal apoptosis [94]. In addition, AS-IV can up-regulate the expression of SIRT1 in tMCAO rats, reduce the levels of acetylated MAPT (ac-MAPT) and phosphorylated MAPT (p-MAPT), and improve neurological deficits after ischemiareperfusion, indicating that AS-IV may play a neuroprotective effect by regulating the SIRT1/MAPT pathway [95].

Galloylpaeoniflorin (GPF) is an effective ingredient extracted from Paeonia lactiflora Pall. or Paeonia veitchii Lynch. GPF pretreatment can increase the levels of phosphorylated Akt and nuclear factor-erythroid 2-related factor 2 (Nrf2) in an OGD cell model, as well as reduce the cerebral infarction volume and improve neurological deficits of rats subjected to cerebral ischemia-reperfusion injury [96].

\subsection{Esters}

Andrographolide is a diterpenoid lactone and it is the main active component of Andrographis paniculata (Burm. f.) Nees. By regulating the Nrf2-HO-1 signaling pathway, andrographolide can inhibit the formation of free radicals and destruction of the BBB in MCAO rats [97].

Ginkgolide B (GB) is a diterpene lactone compound isolated from Ginkgo biloba L. leaves. Pretreatment with GB was shown to down-regulate the expression of Bax, upregulate the expression of Bcl-2, alleviate the damage of neurons and endothelial cells induced by oxygen-glucose deprivation/reoxygenation (OGD/R), and reduce the apoptosis rate of cells [98]. 3-n-butylphthalide (NBP), also known as apigenin, was originally isolated from the seeds of Apium graveolens L., and was shown to protect against acute ischemic stroke [99]. NBP can inhibit the degradation of ATP and reduce the production of ATP-degradation metabolites adenosine, inosine, hypoxanthine and xanthine. Hypoxanthine and xanthine are the substrates that produce oxygen free radicals, suggesting that NBP can reduce the generation of superoxide, reduce lipid peroxidation and neuronal apoptosis [100]. NBP can downregulate the expression of intercellular adhesion molecule-1 (ICAM-1) and protease activated receptor-1 (PAR-1) in cerebral vascular endothelial cells and improve cerebral blood flow after cerebral ischemia-reperfusion in a rat model of tMCAO and photothrombus-induced permanent cerebral ischemia [101].

\subsection{Quinones}

Tanshinone IIA is extracted from the dried rhizome of Salvia miltiorrhiza Bge., and is commonly used in the treatment of cerebrovascular diseases. Tanshinone IIA can promote the recovery of cerebral ischemia-reperfusion injury, increase glucose uptake and energy supply of neurons by targeting GLUT1, and reducing the expression of inflammatory factors COX-2, IL-1 $\beta$, prostaglandin E2 (PGE2) and monocyte chemoattractant protein-1 (MCP-1) [102].

Chrysophanol (CHR) is an active component extracted of Rheum palmatum L. CHR can reduce neuronal damage caused by nitric oxide, decrease the level of 3-nitrotyrosine (3-NT) and the expression of caspase-3 in ischemic brain tissue. It can also increase the activity of manganese dependent superoxide dismutase (MnSOD), interrupt the feedback circuits of ROS/RNS and SOD, and inhibit the production of ROS in ischemic brains, which indicates that CHR can alleviate the nitrification/oxidative stress injury caused by ischemia-reperfusion injury [103]. It has been shown that CHR treatment inhibits GRP78, phosphorylated eukaryotic initiation factor $2 \alpha$ (p-eIF $2 \alpha)$, inhibitory $\kappa \mathrm{B}-\alpha(\mathrm{I} \kappa \mathrm{B}$ $\alpha$ ), CHOP and caspase- 12 in MCAO rats, indicating that CHR protects against ischemia-reperfusion injury by inhibiting endoplasmic reticulum stress-related neuronal apoptosis [104].

\subsection{Terpenoids}

Glycyrrhizin (GL), a triterpenoid isolated from the root of Glycyrrhiza uralensis Fisch., is a natural high mobilitygroupbox 1 (HMGB1) inhibitor. In a rat model of IS, GL can reduce the expression of NADPH oxidase in the brain, inhibit the production of ONOO-, reduce the release of HMGB1, alleviate the $\mathrm{BBB}$ damage and neuronal apoptosis, and reduce the mortality [105].

Carvacrol is a phenolic monoterpene widely found in natural aromatic plants. In a model of focal cerebral ischemia/reperfusion injury in rats, carvacrol could inhibit the level of inflammatory cytokines and the activity of MPO, inhibit the expression of iNOS, cyclooxygenase-2 (COX-2) and nuclear NF- $\kappa \mathrm{B}-\mathrm{p} 65$ as well as increase the activity of SOD 
in ischemic cortical tissues [106]. Carvacrol can be used as a potential drug for the treatment of cerebral ischemic injury.

\subsection{Other compounds}

Trigonelline is a plant alkaloid extracted from the seeds of Trigonella foenum-graecum L., a leguminous plant. Administration of $100 \mathrm{mg} / \mathrm{kg}$ trigonelline immediately after cerebral ischemia in rats can reduce cerebral infarction, improve motor and neurological deficit scores, and significantly inhibit the expression of myeloperoxidase mediated by reduced glutathione in the cerebral cortex [107].

Xyloketal B is a natural compound isolated from mangrove fungi in the South China Sea. It can protect cortical neurons from OGD-induced cell death, reduce the volume of cerebral infarction and improve the recovery of the neural function in rats with hypoxic-ischemic brain injury [108].

Indole-3-carbinol (I3C) is a natural compound with antithrombotic activity extracted from Brassica plants. I3C can increase the levels of SOD and CAT, increase cerebral blood flow, inhibit ADP-induced platelet aggregation, and reduce the volume of ischemic cerebral infarction in $\mathrm{tMCAO}$ rats. In addition, $\mathrm{I} 3 \mathrm{C}$ pretreatment shortened the length of carrageenan-induced tail thrombosis in rats, suggesting its preventive antithrombotic effect [109].

Ergostatrien-7,9(11),22-trien-3 $\beta$-ol (EK100) is the main active ingredient of the ethyl acetate extract of Antrodia camphorata, which has anti-inflammatory and anti-apoptotic effects. EK100 treatment reduced the expression of p65NF$\kappa \mathrm{B}$ and caspase- 3 in the cortex around the infarcted brain area mice subjected to acute ischemic stroke. Meanwhile, EK100 increased the generation of endogenous neural stem cells (DCX+) and the expression level of Bcl2 by activating the PI3K/Akt signaling pathway [110]. The value of EK100 combined with $\mathrm{r}$-tPA in the treatment of human acute ischemic stroke deserves further study.

Achyranthes bidentata polypeptide $\mathrm{K}(\mathrm{ABPPk})$ is an active ingredient isolated from Achyranthes Bidentata Bloom. It can reduce the oxidative damage of brain endothelial cells induced by ischemia, inhibit the expression of tissue factor (TF), plasminogen activator inhibitor-1 (PAI-1) and NF- $\kappa \mathrm{B}$, and reduce downstream microthrombus formation. In addition, ABPPk can inhibit the infiltration of polymorphonuclear leukocytes (PMNs) in the ischemic penumbra, decrease the expression of MMP-2 and MMP-9, prevent the destruction of $\mathrm{BBB}$, and improve neurological function after stroke [111].

\section{Discussion}

The application history of natural compounds in traditional medicine and their excellent safety make them attractive in the development of human disease therapeutics. Clues to the relationship between the intake of natural compounds and beneficial effects on stroke come from epidemiological studies. A prospective study involving 74961 participants showed that the intake of fruits and vegetables was negatively correlated with the risk of stroke, suggesting that phy- tochemicals in fruits and vegetables may reduce the incidence of ischemic stroke by weakening the key pathological processes leading to stroke [114]. In addition, fruit polyphenol supplements have been shown to improve the cognitive and functional recovery of patients with ischemic stroke [115]. Results from a meta-analysis considering 11 prospective cohort studies suggested that high dietary intake of flavonoids may moderately reduce the risk of stroke [116]. Different prospective studies have also examined the relationship between dietary flavonoid subclasses and stroke. Higher dietary flavonol intake has been associated with a reduced risk for stroke [117]. The increased intake of flavanones has been associated with diminished risk of brain ischemia in women [118]. In a cohort study, eating foods rich in quercetin reduced the risk of thrombotic stroke [119]. Pyrogallol, a human metabolite of EGCG in green tea, can inhibit platelet aggregation in human blood, indicating that green tea may play a protective role in brain by inhibiting platelet aggregation [57].

Neuroprotective natural compounds can inhibit the key pathogenic process of ischemic brain injury, and can be used as a powerful candidate for stroke treatment. A growing body of evidence from cell culture models and animal models of brain ischemia suggests the brain protective potential of a variety of natural compounds [120]. Human ischemic stroke is extremely diverse in its manifestations, causes, and anatomic localization, whereas an experimental ischemic stroke is highly reproducible, well controllable, and standardized, allowing more precise analysis of stroke pathophysiology and drug effects, which is an indispensable tool to study stroke [121]. However, there are differences in results between animal models of stroke and humans. The reasons for this difference are: differences in brain function and structure between humans and rodents, difficulty in controlling the time window of clinical treatment after stroke as precisely as in animal experiments and the lack of basic risk factors and pathological processes of the disease in animal models. Long-term, large-scale and well-designed clinical trials are therefore needed.

\section{Conclusions}

Stroke is a common clinical disease of difficult treatment that is characterized by its high morbidity, disability, and mortality. The pathogenesis of stroke is complex, and it involves the regulation of a variety of genes and signaling pathways, which ultimately lead to brain damage and dysfunction. Existing drug therapy or surgical methods have some issues, such as a narrow treatment window, high risk of complications and high failure rate.

Natural products are an important source of drug discovery, being often multi-targeted, regulating multiple pathways, and displaying potential advantages in the treatment of stroke. Natural products can play a neuroprotective role by intervening in excitotoxicity, oxidative and nitrifying stress, inflammation and other pathological processes, thus deserv- 
ing further investigation. The combination of natural products and other treatment methods can synergize to prolong the treatment time window and reduce stroke complications. Therefore, future research should explore the use of natural products in the treatment of stroke. Moreover, it is also necessary to conduct pharmacokinetics, pharmacodynamics and toxicological studies of natural products. Finally, the potential interaction of natural products with rt-PA should be examined before being used as a combination therapy.

\section{Abbreviations}

FDA, Food and Drug Administration; t-PA, tissue plasminogen activator; IS, ischemic; HS, hemorrhagic stroke; iNOS, inducible nitric oxide synthase; Glu, glutamic acid; Asp, aspartic acid; RNS, reactive nitrogen species; NADPH, nicotinamide adenine dinucleotide phosphate; $\mathrm{BBB}$, bloodbrain barrier; TNF- $\alpha$, tumor necrosis factor- $\alpha$; PAF, platelet activating factor; IL- $1 \beta$, interleukin- $\beta$; IL-6, interleukin6 ; IFN- $\gamma$, Interferon- $\gamma$; MMP, matrix metalloproteinase; AQP4, aquaporin 4; MCAO, middle cerebral artery occlusion; PCB, Pinocembrin; LC3II, light chain 3- II; RAPA, rapamycin; CFDA, China Food and Drug Administration; ONOO-, peroxynitrite anion; CaMKII, $\mathrm{Ca}^{2+} /$ calmodulindependent protein kinase II; OGD, oxygen-glucose deprivation; PARP, poly ADP-ribose polymerase; MPO, myeloperoxidases; pMCAO, permanent middle cerebral artery occlusion; iNOS, inducible nitric oxide synthase; tMCAO, cerebral cortex of temporary MCAO; HDAC, histone deacetylase; BDNF, brain-derived neurotrophic factor; Syn-I, synapsin I; Bcl-2, B-cell lymphoma-2; CHOP, CCAAT/enhancer binding protein homologous protein; GRP78, glucose regulatory protein 78; EGCG, Epigallocatechin-3-gallate; OGD/R, oxygen-glucose deprivation/reoxygenation; mTOR, mechanistic target of RAPA; COX-2, cyclooxygenase-2; TGF$\beta$, transforming growth factor- $\beta$; Th17/Tregs, T-helper 17/regulatory $\mathrm{T}$ cells; Th1/Th2, T-helper 1/T-helper 2; GPx, glutathione peroxidase; GR, glutathione reductase; GST, glutathione-S-transferase; SOD, superoxide dismutase; CAT, catalase; RDX6, peroxiredoxin 6; BCCAO, bilateral common carotid arteries occlusion; SalB, Salvianolic acid B; SAA, Salvianolic acid A; AKT, protein kinase B; FKHR, forkhead transcription factor; ERK, extracellular signal-regulated kinase; RASMC, rat arterial smooth muscle cells; Ang1, angiopoietin-1; AS-IV, Astragaloside IV; Cyto C, Cytochrome C; ac-MAPT, acetylated MAPT; p-MAPT, phosphorylated MAPT; GPF, Galloylpaeoniflorin; Nrf2, nuclear factor-erythroid 2-related factor 2; GB, Ginkgolide B; NBP, 3-n-butylphthalide; ICAM-1, intercellular adhesion molecule-1; PAR-1, protease activated receptor-1; PGE2, prostaglandin E2; MCP-1, monocyte chemoattractant protein-1; CHR, chrysophanol; 3-NT, 3-nitrotyrosine; MnSOD, manganese dependent superoxide dismutase; $\mathrm{p}$ eIF $2 \alpha$, phosphorylated eukaryotic initiation factor $2 \alpha$; I $\kappa \mathrm{B}$ $\alpha$, inhibitory $\kappa \mathrm{B}-\alpha$; GL, Glycyrrhizin; HMGB1, high mobility group box 1; I3C, Indole-3-carbinol; EK100,
Ergostatrien-7,9(11),22-trien-3 $\beta$-ol; ABPPk, Achyranthes bidentata polypeptide $\mathrm{K}$; TF, tissue factor; PAI-1, plasminogen activator inhibitor-1; PMNs, polymorphonuclear leukocytes.

\section{Author contributions}

JiaL, TZhao, YL, HQ and MX contributed to literature search; JunL, PS, YF drafted and wrote the article; XWang, CL, TZheng, RC, XWei, YX and JW contributed to the discussion of the review. All authors contributed to editorial changes in the manuscript. All authors read and approved the final manuscript.

\section{Ethics approval and consent to participate}

Not applicable.

\section{Acknowledgment}

We thank three anonymous reviewers for excellent criticism of the article.

\section{Funding}

This study was supported by the National Natural Science Foundation of China (NSFC, Nos. 81874419, 81673719 and 81303074), National Health Commission of the people's Republic of China (No. 2017ZX09301064002) and Youth Innovation Team of Shandong University of Traditional Chinese Medicine.

\section{Conflict of interest}

The authors declare no conflict of interest.

\section{References}

[1] Fukuta T, Asai T, Yanagida Y, Namba M, Koide H, Shimizu K, et al. Combination therapy with liposomal neuroprotectants and tissue plasminogen activator for treatment of ischemic stroke. FASEB Journal. 2017; 31: 1879-1890.

[2] Parrella E, Gussago C, Porrini V, Benarese M, Pizzi M. From preclinical stroke models to humans: Polyphenols in the prevention and treatment of stroke. Nutrients. 2020; 13: 85.

[3] Wu R, Li X, Xu P, Huang L, Cheng J, Huang X, et al. TREM2 protects against cerebral ischemia/reperfusion injury. Molecular Brain. 2017; 10: 20

[4] Campbell BCV, De Silva DA, Macleod MR, Coutts SB, Schwamm LH, Davis SM, et al. Ischaemic stroke. Nature Reviews Disease Primers. 2019; 5: 70.

[5] Wang W, Jiang B, Sun H, Ru X, Sun D, Wang L, et al. Prevalence, Incidence, and Mortality of Stroke in China: Results from a Nationwide Population-Based Survey of 480687 Adults. Circulation. 2017; 135: 759-771.

[6] Rabinstein AA. Update on Treatment of Acute Ischemic Stroke. CONTINUUM: Lifelong Learning in Neurology. 2020; 26: 268 286.

[7] Neuhaus AA, Couch Y, Hadley G, Buchan AM. Neuroprotection in stroke: the importance of collaboration and reproducibility. Brain. 2017; 140: 2079-2092.

[8] Wu J, Wang L, Dong X, Li Z, Wang K, Li L, et al. Chinese herbal injections combined with rt-PA intravenous thrombolysis for acute ischemic stroke. Medicine. 2021; 100: e25004.

[9] Patel RAG, McMullen PW. Neuroprotection in the Treatment of Acute Ischemic Stroke. Progress in Cardiovascular Diseases. 2017; 59: $542-548$. 
[10] Kim J, Fann DY, Seet RCS, Jo D, Mattson MP, Arumugam TV. Phytochemicals in Ischemic Stroke. NeuroMolecular Medicine. 2016; 18: 283-305.

[11] Chen S, Chen H, Du Q, Shen J. Targeting myeloperoxidase (mpo) mediated oxidative stress and inflammation for reducing brain ischemia injury: Potential application of natural compounds. Frontiers in Physiology. 2020; 11: 433.

[12] Subedi L, Gaire BP. Phytochemicals as regulators of microglia/macrophages activation in cerebral ischemia. Pharmacological Research. 2021; 165: 105419.

[13] Chen H, Qi S, Shen J. One-Compound-Multi-Target: Combination Prospect of Natural Compounds with Thrombolytic Therapy in Acute Ischemic Stroke. Current Neuropharmacology. 2017; 15: 134-156.

[14] George PM, Steinberg GK. Novel Stroke Therapeutics: Unraveling Stroke Pathophysiology and its Impact on Clinical Treatments. Neuron. 2015; 87: 297-309.

[15] Kinoshita H, Maki T, Yasuda K, Kishida N, Sasaoka N, Takagi Y, et al. KUS121, a valosin-containing protein modulator, attenuates ischemic stroke via preventing ATP depletion. Scientific Reports. 2019; 9: 11519.

[16] Zhao H, Kilgas S, Alam A, Eguchi S, Ma D. The Role of Extracellular Adenosine Triphosphate in Ischemic Organ Injury. Critical Care Medicine. 2016; 44: 1000-1012.

[17] Khanna A, Kahle KT, Walcott BP, Gerzanich V, Simard JM. Disruption of ion homeostasis in the neurogliovascular unit underlies the pathogenesis of ischemic cerebral edema. Translational Stroke Research. 2014; 5: 3-16.

[18] Zhang M, Gong J, Wang J, Jiang M, Li L, Hu Y, et al. P38 MAPK Participates in the Mediation of GLT-1 up-regulation during the Induction of Brain Ischemic Tolerance by Cerebral Ischemic Preconditioning. Molecular Neurobiology. 2017; 54: 58-71.

[19] Sedlak TW, Paul BD, Parker GM, Hester LD, Snowman AM, Taniguchi $Y$, et al. The glutathione cycle shapes synaptic glutamate activity. Proceedings of the National Academy of Sciences of the United States of America. 2019; 116: 2701-2706.

[20] Rose CR, Felix L, Zeug A, Dietrich D, Reiner A, Henneberger C. Astroglial glutamate signaling and uptake in the hippocampus. Frontiers in Molecular Neuroscience. 2017; 10: 451.

[21] Li LL, Ke XY, Jiang C, Qin SQ, Liu YY, Xian XH, et al. Na+, $\mathrm{K}+-\mathrm{ATPase}$ participates in the protective mechanism of rat cerebral ischemia-reperfusion through the interaction with glutamate transporter-1. Fundamental \& Clinical Pharmacology. 2021; 35: 870-881.

[22] Suárez-Pozos E, Thomason EJ, Fuss B. Glutamate Transporters: Expression and Function in Oligodendrocytes. Neurochemical Research. 2020; 45: 551-560.

[23] Achzet LM, Davison CJ, Shea M, Sturgeon I, Jackson DA. Oxidative stress underlies the ischemia/reperfusion-induced internalization and degradation of ampa receptors. International Journal of Molecular Sciences. 2021; 22: 717.

[24] Duan J, Gao S, Tu S, Lenahan C, Shao A, Sheng J. Pathophysiology and Therapeutic Potential of NADPH Oxidases in Ischemic Stroke-Induced Oxidative Stress. Oxidative Medicine and Cellular Longevity. 2021; 2021: 6631805.

[25] Mi Y, Jiao K, Xu J, Wei K, Liu J, Meng Q, et al. Kellerin from Ferula sinkiangensis exerts neuroprotective effects after focal cerebral ischemia in rats by inhibiting microglia-mediated inflammatory responses. Journal of Ethnopharmacology. 2021; 269: 113718.

[26] Sun S, Chen X, Gao Y, Liu Z, Zhai Q, Xiong L, et al. Mn-SOD Upregulation by Electroacupuncture Attenuates Ischemic Oxidative Damage via CB1R-Mediated STAT3 Phosphorylation. Molecular Neurobiology. 2016; 53: 331-343.

[27] Sun M, Jin H, Sun X, Huang S, Zhang F, Guo Z, et al. Free Radical Damage in Ischemia-Reperfusion Injury: an Obstacle in Acute Ischemic Stroke after Revascularization Therapy. Oxidative Medicine and Cellular Longevity. 2018; 2018: 3804979.

[28] Qin C, Zhou L, Ma X, Hu Z, Yang S, Chen M, et al. Dual Functions of Microglia in Ischemic Stroke. Neuroscience Bulletin. 2019; 35:
921-933.

[29] Xu S, Lu J, Shao A, Zhang JH, Zhang J. Glial cells: Role of the immune response in ischemic stroke. Frontiers in Immunology. 2020; 11: 294.

[30] Scimemi A. Astrocytes and the Warning Signs of Intracerebral Hemorrhagic Stroke. Neural Plasticity. 2018; 2018: 7301623.

[31] Xu H, Qin W, Hu X, Mu S, Zhu J, Lu W, et al. Lentivirus-mediated overexpression of OTULIN ameliorates microglia activation and neuroinflammation by depressing the activation of the NF- $x \mathrm{~B}$ signaling pathway in cerebral ischemia/reperfusion rats. Journal of Neuroinflammation. 2018; 15: 83.

[32] Acosta SA, Lee JY, Nguyen H, Kaneko Y, Borlongan CV. Endothelial Progenitor Cells Modulate Inflammation-Associated Stroke Vasculome. Stem Cell Reviews and Reports. 2019; 15: 256-275.

[33] Gupta R, Ghosh S. Putative roles of mitochondrial VoltageDependent Anion Channel, Bcl-2 family proteins and c-Jun $\mathrm{N}$-terminal Kinases in ischemic stroke associated apoptosis. Biochimie Open. 2017; 4: 47-55.

[34] Qi Z, Dong W, Shi W, Wang R, Zhang C, Zhao Y, et al. Bcl2 phosphorylation triggers autophagy switch and reduces mitochondrial damage in limb remote ischemic conditioned rats after ischemic stroke. Translational Stroke Research. 2015; 6: 198-206.

[35] Sun D, Xu M, Chen Q, Hu H. Significant elevation of serum caspase-3 levels in patients with intracerebral hemorrhage. Clinica Chimica Acta. 2017; 471: 62-67.

[36] Wang X, Luo Y, Sun H, Feng J, Ma S, Liu J, et al. Dynamic expression changes of Bcl-2, Caspase- 3 and Hsp70 in middle cerebral artery occlusion rats. Brain Injury. 2015; 29: 93-97.

[37] Zhang T, Wang H, Li Q, Fu J, Huang J, Zhao Y. MALAT1 Activates the P53 Signaling Pathway by Regulating MDM2 to Promote Ischemic Stroke. Cellular Physiology and Biochemistry. 2018; 50: 2216-2228.

[38] Radak D, Katsiki N, Resanovic I, Jovanovic A, Sudar-Milovanovic E, Zafirovic S, et al. Apoptosis and Acute Brain Ischemia in Ischemic Stroke. Current Vascular Pharmacology. 2017; 15: 115122.

[39] Jiang MQ, Zhao Y, Cao W, Wei ZZ, Gu X, Wei L, et al. Longterm survival and regeneration of neuronal and vasculature cells inside the core region after ischemic stroke in adult mice. Brain Pathology. 2017; 27: 480-498.

[40] Jiang X, Andjelkovic AV, Zhu L, Yang T, Bennett MVL, Chen J, et al. Blood-brain barrier dysfunction and recovery after ischemic stroke. Progress in Neurobiology. 2018; 163-164: 144-171.

[41] Yang C, Hawkins KE, Doré S, Candelario-Jalil E. Neuroinflammatory mechanisms of blood-brain barrier damage in ischemic stroke. American Journal of Physiology-Cell Physiology. 2019; 316: C135-C153.

[42] Shi Z, Fang Q, Chen Y, Xu L, Wu M, Jia M, et al. Methylene blue ameliorates brain edema in rats with experimental ischemic stroke via inhibiting aquaporin 4 expression. Acta Pharmacologica Sinica. 2021; 42: 382-392.

[43] Sylvain NJ, Salman MM, Pushie MJ, Hou H, Meher V, Herlo R, et al. The effects of trifluoperazine on brain edema, aquaporin-4 expression and metabolic markers during the acute phase of stroke using photothrombotic mouse model. Biochimica Et Biophysica Acta (BBA) - Biomembranes. 2021; 1863: 183573.

[44] Huang L, Shang E, Fan W, Li X, Li B, He S, et al. S-oxiracetam protect against ischemic stroke via alleviating blood brain barrier dysfunction in rats. European Journal of Pharmaceutical Sciences. 2017; 109: 40-47.

[45] Wu S, Yuan R, Wang Y, Wei C, Zhang S, Yang X, et al. Early Prediction of Malignant Brain Edema after Ischemic Stroke. Stroke. 2018; 49: 2918-2927.

[46] Wang J, Mei Z, Fu Y, Yang S, Zhang S, Huang W, et al. Puerarin protects rat brain against ischemia/reperfusion injury by suppressing autophagy via the AMPK-mTOR-ULK1 signaling pathway. Neural Regeneration Research. 2018; 13: 989-998.

[47] Hongyun H, Tao G, Pengyue Z, Liqiang Y, Yihao D. Puerarin provides a neuroprotection against transient cerebral ischemia by at- 
tenuating autophagy at the ischemic penumbra in neurons but not in astrocytes. Neuroscience Letters. 2017; 643: 45-51.

[48] Zheng Q, Li X, Mei Z, Xiong L, Mei Q, Wang J, et al. Efficacy and safety of puerarin injection in curing acute ischemic stroke: a meta-analysis of randomized controlled trials. Medicine. 2017; 96: e5803.

[49] Ma Y, Li L, Kong L, Zhu Z, Zhang W, Song J, et al. Pinocembrin Protects Blood-Brain Barrier Function and Expands the Therapeutic Time Window for Tissue-Type Plasminogen Activator Treatment in a Rat Thromboembolic Stroke Model. BioMed Research International. 2018; 2018: 8943210.

[50] Tao J, Shen C, Sun Y, Chen W, Yan G. Neuroprotective effects of pinocembrin on ischemia/reperfusion-induced brain injury by inhibiting autophagy. Biomedicine \& Pharmacotherapy. 2018; 106: 1003-1010.

[51] Shen X, Liu Y, Luo X, Yang Z. Advances in biosynthesis, pharmacology, and pharmacokinetics of pinocembrin, a promising natural small-molecule drug. Molecules. 2019; 24.

[52] Chen H, Guan B, Chen X, Chen X, Li C, Qiu J, et al. Baicalin Attenuates Blood-Brain Barrier Disruption and Hemorrhagic Transformation and Improves Neurological Outcome in Ischemic Stroke Rats with Delayed t-PA Treatment: Involvement of ONOOMMP-9 Pathway. Translational Stroke Research. 2018; 9: 515529.

[53] Wang P, Cao Y, Yu J, Liu R, Bai B, Qi H, et al. Baicalin alleviates ischemia-induced memory impairment by inhibiting the phosphorylation of CaMKII in hippocampus. Brain Research. 2016; 1642: 95-103.

[54] PARK D, SHAH F, KOH P. Quercetin attenuates neuronal cells damage in a middle cerebral artery occlusion animal model. Journal of Veterinary Medical Science. 2018; 80: 676-683.

[55] Annapurna A, Ansari MA, Manjunath PM. Partial role of multiple pathways in infarct size limiting effect of quercetin and rutin against cerebral ischemia-reperfusion injury in rats. European Review for Medical and Pharmacological Sciences. 2013; 17: 491500.

[56] Park D, Kang J, Shah F, Koh P. Quercetin attenuates the reduction of parvalbumin in middle cerebral artery occlusion animal model. Laboratory Animal Research. 2021; 37: 9.

[57] Applová L, Karlíčková J, Warncke P, Macáková K, Hrubša M, Macháček M, et al. 4-Methylcatechol, a Flavonoid Metabolite with Potent Antiplatelet Effects. Molecular Nutrition \& Food Research. 2019; 63: 1900261.

[58] Ferreira EDO, Fernandes MYSD, Lima NMRD, Neves KRT, Carmo MRSD, Lima FAV, et al. Neuroinflammatory response to experimental stroke is inhibited by eriodictyol. Behavioural Brain Research. 2016; 312: 321-332.

[59] Wang C, Ma Z, Wang Z, Ming S, Ding Y, Zhou S, et al. Eriodictyol attenuates mcao-induced brain injury and neurological deficits via reversing the autophagy dysfunction. Frontiers in Systems Neuroscience. 2021; 15: 655125.

[60] Zhao J, Song J, Pan S, Wang K. Treatment with Isorhamnetin Protects the Brain against Ischemic Injury in Mice. Neurochemical Research. 2016; 41: 1939-1948.

[61] Tu F, Pang Q, Huang T, Zhao Y, Liu M, Chen X. Apigenin Ameliorates Post-Stroke Cognitive Deficits in Rats through Histone Acetylation-Mediated Neurochemical Alterations. Medical Science Monitor. 2017; 23: 4004-4013.

[62] Pang Q, Zhao Y, Chen X, Zhao K, Zhai Q, Tu F. Apigenin Protects the Brain against Ischemia/Reperfusion Injury via Caveolin1/VEGF in Vitro and in Vivo. Oxidative Medicine and Cellular Longevity. 2018; 2018: 1-12.

[63] Feng J, Chen X, Lu S, Li W, Yang D, Su W, et al. Naringin Attenuates Cerebral Ischemia-Reperfusion Injury through InhibitingPeroxynitrite-Mediated Mitophagy Activation. Molecular Neurobiology. 2018; 55: 9029-9042.

[64] Okuyama S, Yamamoto K, Mori H, Sawamoto A, Amakura Y, Yoshimura M, et al. Neuroprotective effect of Citrus kawachiensis (Kawachi Bankan) peels, a rich source of naringin, against global cerebral ischemia/reperfusion injury in mice. Bioscience, Biotechnology, and Biochemistry. 2018; 82: 1216-1224.

[65] Okuyama S, Katoh M, Kanzaki T, Kotani Y, Amakura Y, Yoshimura M, et al. Auraptene/Naringin-Rich Fruit Juice of Citrus kawachiensis (Kawachi Bankan) Prevents Ischemia-Induced Neuronal Cell Death in Mouse Brain through Anti-Inflammatory Responses. Journal of Nutritional Science and Vitaminology. 2019; 65: 66-71.

[66] Yang J, Yuan L, Wen Y, Zhou H, Jiang W, Xu D, et al. Protective effects of naringin in cerebral infarction and its molecular mechanism. Medical Science Monitor. 2020; 26: e918772.

[67] Akbari-Kordkheyli V, Abbaszadeh-Goudarzi K, NejatiLaskokalayeh M, Zarpou S, Khonakdar-Tarsi A. The protective effects of silymarin on ischemia-reperfusion injuries: a mechanistic review. Iranian Journal of Basic Medical Sciences. 2019; 22: 968-976.

[68] Wang M, Li Y, Ding Y, Zhang H, Sun T, Zhang K, et al. Silibinin Prevents Autophagic Cell Death upon Oxidative Stress in Cortical Neurons and Cerebral Ischemia-Reperfusion Injury. Molecular Neurobiology. 2016; 53: 932-943.

[69] Wang M, Chen Z, Yang L, Ding L. Sappanone A Protects against Inflammation, Oxidative Stress and Apoptosis in Cerebral Ischemia-Reperfusion Injury by Alleviating Endoplasmic Reticulum Stress. Inflammation. 2021; 44: 934-945.

[70] Nan W, Zhonghang X, Keyan C, Tongtong L, Wanshu G, Zhongxin X. Epigallocatechin-3-Gallate Reduces Neuronal Apoptosis in Rats after Middle Cerebral Artery Occlusion Injury via PI3K/AKT/eNOS Signaling Pathway. BioMed Research International. 2018; 2018: 6473580.

[71] Wang X, You Y. Epigallocatechin Gallate Extends Therapeutic Window of Recombinant Tissue Plasminogen Activator Treatment for Brain Ischemic Stroke: a Randomized Double-Blind and Placebo-Controlled Trial. Clinical Neuropharmacology. 2017; 40: 24-28.

[72] Bai Q, Lyu Z, Yang X, Pan Z, Lou J, Dong T. Epigallocatechin-3gallate promotes angiogenesis via up-regulation of $\mathrm{Nfr} 2$ signaling pathway in a mouse model of ischemic stroke. Behavioural Brain Research. 2017; 321: 79-86.

[73] Zálešák F, Bon DJD, Pospíšil J. Lignans and Neolignans: Plant secondary metabolites as a reservoir of biologically active substances. Pharmacological Research. 2019; 146: 104284.

[74] Peterson J, Dwyer J, Adlercreutz H, Scalbert A, Jacques P, McCullough ML. Dietary lignans: physiology and potential for cardiovascular disease risk reduction. Nutrition Reviews. 2010; 68: 571603.

[75] Wang G, Wang T, Zhang Y, Li F, Yu B, Kou J. Schizandrin protects against $\mathrm{OGD} / \mathrm{R}$-induced neuronal injury by suppressing autophagy: Involvement of the ampk/mtor pathway. Molecules. $2019 ; 24$.

[76] Zhou F, Wang M, Ju J, Wang Y, Liu Z, Zhao X, et al. Schizandrin A protects against cerebral ischemia-reperfusion injury by suppressing inflammation and oxidative stress and regulating the AMPK/Nrf2 pathway regulation. American Journal of Translational Research. 2019; 11: 199-209.

[77] Reinisalo M, Kårlund A, Koskela A, Kaarniranta K, Karjalainen RO. Polyphenol Stilbenes: Molecular Mechanisms of Defence against Oxidative Stress and Aging-Related Diseases. Oxidative Medicine and Cellular Longevity. 2015; 2015: 340520.

[78] Benakis C, Brea D, Caballero S, Faraco G, Moore J, Murphy M, et al. Commensal microbiota affects ischemic stroke outcome by regulating intestinal $\gamma \delta$ T cells. Nature Medicine. 2016; 22: 516523.

[79] Dou Z, Rong X, Zhao E, Zhang L, Lv Y. Neuroprotection of Resveratrol against Focal Cerebral Ischemia/Reperfusion Injury in Mice through a Mechanism Targeting Gut-Brain Axis. Cellular and Molecular Neurobiology. 2019; 39: 883-898.

[80] Ashafaq M, Intakhab Alam M, Khan A, Islam F, Khuwaja G, Hussain $S$, et al. Nanoparticles of resveratrol attenuates oxidative stress and inflammation after ischemic stroke in rats. International Im- 
munopharmacology. 2021; 94: 107494.

[81] Fodor K, Tit DM, Pasca B, Bustea C, Uivarosan D, Endres L, et al. Long-Term Resveratrol Supplementation as a Secondary Prophylaxis for Stroke. Oxidative Medicine and Cellular Longevity. 2018; 2018: 4147320.

[82] Jia G, Tan B, Ma J, Zhang L, Jin X, Li C. Prdx6 Upregulation by Curcumin Attenuates Ischemic Oxidative Damage via SP1 in Rats after Stroke. BioMed Research International. 2017; 2017: 6597401.

[83] Yang X, Song D, Chen L, Xiao H, Ma X, Jiang Q, et al. Curcumin promotes neurogenesis of hippocampal dentate gyrus via Wnt/ $\beta$ catenin signal pathway following cerebral ischemia in mice. Brain Research. 2021; 1751: 147197.

[84] Zhao B, Shi Q, Zhang Z, Wang S, Wang X, Wang H. Protective effects of paeonol on subacute/chronic brain injury during cerebral ischemia in rats. Experimental and Therapeutic Medicine. 2018; 15: 3836-3846.

[85] Wang Y, Chen G, Yu X, Li Y, Zhang L, He Z, et al. Salvianolic Acid B Ameliorates Cerebral Ischemia/Reperfusion Injury through Inhibiting TLR4/MyD88 Signaling Pathway. Inflammation. 2016; 39: 1503-1513.

[86] Fan Y, Luo Q, Wei J, Lin R, Lin L, Li Y, et al. Mechanism of salvianolic acid $B$ neuroprotection against ischemia/reperfusion induced cerebral injury. Brain Research. 2018; 1679: 125-133.

[87] Kapoor M, Sharma N, Sandhir R, Nehru B. Effect of the NADPH oxidase inhibitor apocynin on ischemia-reperfusion hippocampus injury in rat brain. Biomedicine \& Pharmacotherapy. 2018; 97: 458-472.

[88] Qin YY, Li M, Feng X, Wang J, Cao L, Shen XK, et al. Combined NADPH and the NOX inhibitor apocynin provides greater anti-inflammatory and neuroprotective effects in a mouse model of stroke. Free Radical Biology and Medicine. 2017; 104: 333-345.

[89] Feng S, Aa N, Geng J, Huang J, Sun R, Ge C, et al. Pharmacokinetic and metabolomic analyses of the neuroprotective effects of salvianolic acid a in a rat ischemic stroke model. Acta Pharmacologica Sinica. 2017; 38: 1435-1444.

[90] Jiao CX, Zhou H, Yang CX, Ma C, Yang YX, Mao RR, et al. Protective efficacy of a single salvianolic acid a treatment on photothrombosis-induced sustained spatial memory impairments. Neuropsychiatric Disease and Treatment. 2017; 13: 1181-1192.

[91] Mahmood Q, Wang G, Wu G, Wang H, Zhou C, Yang H, et al. Salvianolic acid a inhibits calpain activation and eNOS uncoupling during focal cerebral ischemia in mice. Phytomedicine. 2017; 25: 8-14.

[92] Kang Z, Jiang W, Luan H, Zhao F, Zhang S. Cornin induces angiogenesis through PI3K-Akt-eNOS-VEGF signaling pathway. Food and Chemical Toxicology. 2013; 58: 340-346.

[93] Xu Y, Zhang G, Kang Z, Xu Y, Jiang W, Zhang S. Cornin increases angiogenesis and improves functional recovery after stroke via the ang1/tie 2 axis and the $\mathrm{Wnt} / \beta$-catenin pathway. Archives of Pharmacal Research. 2016; 39: 133-142.

[94] Yin F, Zhou H, Fang Y, Li C, He Y, Yu L, et al. Astragaloside IV alleviates ischemia reperfusion-induced apoptosis by inhibiting the activation of key factors in death receptor pathway and mitochondrial pathway. Journal of Ethnopharmacology. 2020; 248: 112319.

[95] Sin F, Zhou H, Fang Y, Li C, He Y, Yu L, et al. Astragaloside IV alleviates ischemia reperfusion-induced apoptosis by inhibiting the activation of key factors in death receptor pathway and mitochondrial pathway. Journal of Ethnopharmacology. 2020; 248: 112319.

[96] Wen Z, Hou W, Wu W, Zhao Y, Dong X, Bai X, et al. 6'-O-Galloylpaeoniflorin Attenuates Cerebral Ischemia Reperfusion-Induced Neuroinflammation and Oxidative Stress via PI3K/Akt/Nrf2 Activation. Oxidative Medicine and Cellular Longevity. 2018; 2018: 8678267.

[97] Yen T, Chen R, Jayakumar T, Lu W, Hsieh C, Hsu M, et al. Andrographolide stimulates p38 mitogen-activated protein kinasenuclear factor erythroid-2-related factor 2-heme oxygenase 1 signaling in primary cerebral endothelial cells for definite protection against ischemic stroke in rats. Translational Research. 2016; 170:
57-72.

[98] Yang X, Zheng T, Hong H, Cai N, Zhou X, Sun C, et al. Neuroprotective effects of Ginkgo biloba extract and Ginkgolide B against oxygen-glucose deprivation/reoxygenation and glucose injury in a new in vitro multicellular network model. Frontiers of Medicine. 2018; 12: 307-318.

[99] Zhou P, Wang L, Qu M, Shen H, Zheng H, Deng L, et al. Dl-3-N-butylphthalide promotes angiogenesis and upregulates sonic hedgehog expression after cerebral ischemia in rats. CNS Neuroscience \& Therapeutics. 2019; 25: 748-758.

[100] Liu R, Fan C, Zhang Z, Zhao X, Sun Y, Liu H, et al. Effects of Dl-3$\mathrm{n}$-butylphthalide on Cerebral Ischemia Infarction in Rat Model by Mass Spectrometry Imaging. International Journal of Molecular Sciences. 2017; 18: 2451.

[101] Yang C, Guo A, Li Y, Shi K, Shi F, Li M. Dl-3-n-butylphthalide Reduces Neurovascular Inflammation and Ischemic Brain Injury in Mice. Aging and Disease. 2019; 10: 964.

[102] Wang J, Tong H, Wang X, Wang X, Wang Y. Tanshinone IIA alleviates the damage of neurocytes by targeting GLUT1 in ischaemia reperfusion model (in vivo and in vitro experiments). Folia Neuropathologica. 2020; 58: 176-193.

[103] Zhao Y, Huang Y, Fang Y, Zhao H, Shi W, Li J, et al. Chrysophanol attenuates nitrosative/oxidative stress injury in a mouse model of focal cerebral ischemia/reperfusion. Journal of Pharmacological Sciences. 2018; 138: 16-22.

[104] Zhao Y, Fang Y, Zhao H, Li J, Duan Y, Shi W, et al. Chrysophanol inhibits endoplasmic reticulum stress in cerebral ischemia and reperfusion mice. European Journal of Pharmacology. 2018; 818: $1-9$.

[105] Chen H, Guan B, Wang B, Pu H, Bai X, Chen X, et al. Glycyrrhizin Prevents Hemorrhagic Transformation and Improves Neurological Outcome in Ischemic Stroke with Delayed Thrombolysis through Targeting Peroxynitrite-Mediated HMGB1Signaling. Translational Stroke Research. 2020; 11: 967-982.

[106] Li Z, Hua C, Pan X, Fu X, Wu W. Carvacrol Exerts Neuroprotective Effects via Suppression of the Inflammatory Response in Middle Cerebral Artery Occlusion Rats. Inflammation. 2016; 39: 1566-1572.

[107] Pravalika K, Sarmah D, Kaur H, Vats K, Saraf J, Wanve M, et al. Trigonelline therapy confers neuroprotection by reduced glutathione mediated myeloperoxidase expression in animal model of ischemic stroke. Life Sciences. 2019; 216: 49-58.

[108] Xiao A, Chen W, Xu B, Liu R, Turlova E, Barszczyk A, et al. Marine compound xyloketal B reduces neonatal hypoxic-ischemic brain injury. Marine Drugs. 2014; 13: 29-47.

[109] Paliwal P, Chauhan G, Gautam D, Dash D, Patne SCU, Krishnamurthy $S$. Indole-3-carbinol improves neurobehavioral symptoms in a cerebral ischemic stroke model. Naunyn-Schmiedeberg's Archives of Pharmacology. 2018; 391: 613-625.

[110] Wang YH, Chern CM, Liou KT, Kuo YH, Shen YC. Ergostatrien-7, 9 (11), 22-trien-3 $\beta$-ol from Antrodia camphorate ameliorates ischemic stroke brain injury via downregulation of p65NF- $x-\mathrm{B}$ and caspase 3, and activation of Akt/GSK3/cateninassociated neurogenesis. Food \& Function. 2019; 10: 4725-4738.

[111] Cheng Q, Tong F, Shen Y, He C, Wang C, Ding F. Achyranthes bidentata polypeptide $\mathrm{k}$ improves long-term neurological outcomes through reducing downstream microvascular thrombosis in experimental ischemic stroke. Brain Research. 2019; 1706: 166176.

[112] Tressera-Rimbau A, Arranz S, Eder M, Vallverdú-Queralt A. Dietary Polyphenols in the Prevention of Stroke. Oxidative Medicine and Cellular Longevity. 2017; 2017: 7467962.

[113] Tresserra-Rimbau A, Lamuela-Raventos RM, Moreno JJ. Polyphenols, food and pharma. Current knowledge and directions for future research. Biochemical Pharmacology. 2018; 156: 186-195.

[114] Larsson SC, Virtamo J, Wolk A. Total and specific fruit and vegetable consumption and risk of stroke: a prospective study. Atherosclerosis. 2013; 227: 147-152. 
[115] Bellone JA, Murray JR, Jorge P, Fogel TG, Kim M, Wallace DR, et al. Pomegranate supplementation improves cognitive and functional recovery following ischemic stroke: a randomized trial. $\mathrm{Nu}-$ tritional Neuroscience. 2019; 22: 738-743.

[116] Tang Z, Li M, Zhang X, Hou W. Dietary flavonoid intake and the risk of stroke: a dose-response meta-analysis of prospective cohort studies. BMJ Open. 2016; 6: e008680.

[117] Wang Z, Zhao D, Nie Z, Zhao H, Zhou B, Gao W, et al. Flavonol intake and stroke risk: a meta-analysis of cohort studies. Nutrition. 2014; 30: 518-523.

[118] Cassidy A, Rimm EB, O’Reilly EJ, Logroscino G, Kay C, Chiuve $\mathrm{SE}$, et al. Dietary flavonoids and risk of stroke in women. Stroke.
2012; 43: 946-951.

[119] Knekt P, Isotupa S, Rissanen H, Heliövaara M, Järvinen R, Häkkinen $S$, et al. Quercetin intake and the incidence of cerebrovascular disease. European Journal of Clinical Nutrition. 2000; 54: 415417.

[120] Gaire BP. Herbal Medicine in Ischemic Stroke: Challenges and Prospective. Chinese Journal of Integrative Medicine. 2018; 24: 243-246.

[121] Fluri F, Schuhmann MK, Kleinschnitz C. Animal models of ischemic stroke and their application in clinical research. Drug Design, Development and Therapy. 2015; 9: 3445-3454. 